Nuclear Physics A444 (1985) 541-578

(C) North-Holland Publishing Company

\title{
THE NN INTERACTION IN A QUARK MODEL WITH QUARK-ANTIQUARK EXCITATIONS
}

\author{
Y. FUJIWARA and K.T. HECHT \\ Physics Department, University of Michigan, Ann Arbor, Michigan 48109, USA ${ }^{1}$
}

Received 15 April 1985

\begin{abstract}
Quark-antiquark excitations inherent in the quark-gluon interaction have been incorporated into a quark model of the nucleon to study the effects of such excitations on the NN interaction within the framework of the resonating group method. The three-quark $(3 q)$ components of a single nucleon are augmented by (3q)( $q \bar{q})$ excitations with the 24 possible spin, isospin, color combinations for the energetically lowest p-wave relative motion function. Quark exchange kernels are then calculated for the two-nucleon system described by these improved nucleon internal functions; and these exchange kernels are converted to phase-shift-equivalent effective $N N$ potentials by the Wigner-transform WKB technique. The off-shell $q \bar{q}$ pair-creation terms are derived from the one-gluon exchange diagrams in the Breit approximation in analogy with the derivation of the qq and $q \bar{q}$ potentials. The parameters of the interaction are chosen to be consistent with the experimental $\Delta-\mathrm{N}$ mass difference, the nucleon-vector-meson coupling constants, and the nucleon magnetic moments. Within these constraints, the predicted amplitudes of the $(3 q)(q \bar{q})$ components of the nucleon internal functions have been shown to be insensitive to the precise values of the model parameters. In particular, they pass the crucial test of being insensitive to very large changes in the magnitude of the confinement potential constant which is a necessary ingredient of the model. The $q \bar{q}$ excitations lead to the following effects in the S-wave NN potentials: (i) The repulsive core heights of the simple $3 q-3 q$ model are greatly reduced but retain their strong essentially linear energy dependence, with numerical values very similar to those of the short-range phenomenological terms of the Paris potential. (ii) The effective potentials have acquired an attractive part in the $0.8-1.5 \mathrm{fm}$ range. However, this attraction is too weak to bind the deuteron or fit the extreme low-energy S-wave phase shifts.
\end{abstract}

\section{Introduction}

Since quantum chromodynamics (QCD) is believed to be the fundamental theory of the strong interaction, there have been many recent attempts to understand the structure of the nucleon and the NN interaction ${ }^{1-24}$ ) in terms of QCD-inspired, phenomenological quark models. For low-energy nuclear phenomena the gluon degrees of freedom are eliminated from the theoretical framework, and it is assumed that the gluon exchange effects can be incorporated into the theory through a gluon exchange potential, usually in a one-gluon cxchange approximation, through the color analog of the Fermi-Breit interaction. In these constituent quark models or potential models of hadronic structure the dressed non-strange quarks are assumed to have a mass approximately one-third of the mass of the nucleon. The various

'Supported by the US National Science Foundation. 
quark models differ mainly in the phenomenology used to introduce quark confinement. In the potential models confinement is usually achieved through a simple power-law confining potential which is put into the theory by hand.

Of the various techniques used to convert the interaction among quarks into an effective NN interaction potential, the resonating group method (RGM) has the great advantage that the quark exchange kernels needed can be evaluated in very explicit analytic form. A number of detailed RGM and other calculations have been undertaken in an attempt to elucidate the extreme short-range part of the NN interaction $\left.{ }^{3-19}\right)$. In these calculations the nucleon is assumed to be a simple threequark (3q) system with a spatial $3 q$ wave function of $(0 s)^{3}$ character, usually of harmonic-oscillator (gaussian) radial form. The conclusions of such calculations can be summarized as follows: The phase-shift equivalent effective NN potentials derived from the $3 q-3 q$ models of $N N$ scattering are short range, strongly energy dependent, and purely repulsive. The repulsive core of several hundred $\mathrm{MeV}$ is determined largely by the color-magnetic contact term of the Breit interaction. [A recent study ${ }^{16}$ ) shows that the Breit retardation or Darwin-like momentum dependent terms in the color interaction may play a significant role in reducing this core height to around $400 \mathrm{MeV}$.] The coupling to other channels of the $3 \mathrm{q}-3 \mathrm{q}$ variety such as the $\Delta-\Delta$ and the so-called hidden color channels ${ }^{10}$ ) has been studied in detail. Although the coupling to hidden color channels leads to a slight decrease in the repulsive character of the S-wave phase shifts ${ }^{8,11,17,19}$ ), the channel coupling does not play a prominent role and only leads to small refinements. In addition, the repulsive cores are remarkably insensitive to a wide range of parameters of the quark potential models provided their choice is consistent with the single-baryon physics. The essential parameters of such models are four in number: the constituent quark mass $m$, the size parameter $b$ (e.g. an oscillator length parameter) of the 0 s quark wave function, $\alpha_{\mathrm{s}}$ the QCD analog of the fine structure constant (in principle governed by the running strong-coupling constant relevant for nuclear energies), and a potential constant $a_{\mathrm{c}}$ of the phenomenological confinement potential. The parameters $m$ and $b$ are strongly constrained by the electromagnetic moments and the size of the nucleon (although the nucleon size may serve only as an upper bound for $b$ since a pure $3 \mathrm{q}$ picture of the nucleon may describe only the inner core of the nucleon). The parameter $\alpha_{\mathrm{s}}$ is determined by the $\Delta-\mathrm{N}$ mass difference, through the combination $\alpha_{\mathrm{s}} / \mathrm{m}^{2} b^{3}$. The confinement potential constant is determined either by fitting the nucleon mass or through the stability condition which minimizes the nucleon mass to changes in $b$. The importance of this stability condition has been stressed ${ }^{23}$ ) in calculations in which breathing-mode excitations for the $3 q$ wave functions are included in the basis, but it becomes significant for the choice of parameters only if such excitations are part of the RGM nucleon internal functions.

The almost complete insensitivity of the repulsive cores to the confinement potential may come as somewhat of a surprise. The quark exchange kernel generated 
by a purely quadratic confinement potential is proportional to the norm kernel ${ }^{7,13}$ ). The scattering phase shifts are therefore completely independent of the strength of this potential ${ }^{7}$ ). A linear confinement potential gives only a very small contribution to the repulsive core in the NN interaction ${ }^{16}$ ), and final results for the NN scattering phase shifts are very insensitive to large changes in the strengths of the confining potential. This conclusion is valid only for a properly constrained model space. If both $\mathrm{p}$-wave and color-octet excitations in the $3 \mathrm{q}$ internal wave functions are permitted, the resultant color deformability leads to long-range Van der Waals potentials [proportional to the strength of the confinement potential with an $R^{n-4}$ power-law dependence ${ }^{25}$ ), where $R$ is the NN separation and $n$ is the exponent of the confining potential]. We consider it one of the advantages of the RGM method that such pathological effects are automatically excluded by a proper choice of the RGM nucleon internal wave functions. In a recent quark-model study of the NN interaction, Maltman and Isgur ${ }^{18}$ ) predict a seemingly realistic medium-range attraction. Since their model space includes excitations of this color-deformable type it appears that this attraction has its origin in the confining potential and also leads to an unrealistic long-range power-law attraction of color Van der Waals type. A weak medium-range attraction is also predicted by Robson ${ }^{24}$ ) in a quark-model calculation in which $2 \hbar \omega$ excitations of mixed orbital [21] symmetry are included in the $3 q$ internal wave functions. Although these do not lead to Van der Waals contaminations it is not clear what mechanism gives rise to this attraction.

Due to the phenomenological character of the confinement potential, it is the philosophy of the present investigation that any nucleon property which is strongly dependent on the strength of the confinement potential cannot be trusted. Since a properly constrained RGM model space gives an NN potential which is very insensitive to the strength and form of the confining potential, the results of the present investigation are free of this difficulty. In addition, the predicted $\Delta-\mathrm{N}$ mass difference and the electromagnetic properties of the nucleon (which are used to fix the parameters $\alpha_{s}, m$ and $b$ ) are also insensitive to the strength of the confining potential.

One of the disadvantages of the RGM and quark potential model calculations is that these are essentially nonrelativistic. The parameter $x=\hbar / m c b$ (ratio of quark Compton wavelength to size parameter $b$ ) gives a measure of the importance of relativistic effects. Since $x \sim 1$ in most recent quark potential model studies (in the present investigation the final choice is $x=0.8$ ), it appears that a nonrelativistic treatment is out of the question. For absolute predictions of single-baryon masses a fully relativistic treatment is surely required. However, the relative motion of two nucleons is essentially nonrelativistic, and it is encouraging to note that a recent study of relativistic kinetic energy corrections to the $3 q-3 q$ exchange kernels gives only a small contribution to the NN repulsive core, less than $10 \%$ of that of the dominant color-magnetic contribution ${ }^{16}$ ). Due to the difficulties of a fully relativistic treatment of the $3 q-3 q$ system the quark potential models usually neglect terms of 
order $x^{4}$ and retain only the "dominant" $x^{2}$ terms in the color Breit interaction. It must therefore also be assumed that the parameters $\alpha_{\mathrm{s}}, m, b$ and $a_{\mathrm{c}}$ are renormalized parameters which carry some of the burden of relativistic corrections. Predictions of the electromagnetic properties of the nucleon, which are used in the fit of parameters in the present investigation, will, however, make use of relativistic electromagnetic current operators of quarks and antiquarks. In this case the use of nonrelativistic wave functions is to be considered in the spirit of a Foldy-Wouthuysen treatment.

One of the greatest drawbacks of the 3q-3q potential models of $\mathrm{NN}$ scattering comes from the fact that they give only the extreme short-range part of the NN interaction and fail to make a natural connection to the mesonic degrees of freedom responsible for the medium- and long-range attractive part of this interaction. In contrast, in the little or chiral bag models ${ }^{26}$ ) or the cloudy bag models ${ }^{27}$ ) the quark degrees of freedom of the nucleon interior are coupled to an exterior meson field. More recent models of nucleon structure are an outgrowth of the Friedberg-Lee model ${ }^{28}$ ) in which the quarks are coupled to a scalar field which confines the quarks to the finite nucleon interior. In later versions of this model the quarks are coupled to both a confining field and the more conventional meson fields of nuclear structure, leading to various nontopological soliton models ${ }^{29}$ ). Models which contain both quarks and meson fields but have some of the interesting topological properties of the topological soliton or skyrmion models ${ }^{30}$ ) have also been proposed ${ }^{31}$ ). However, the emphasis in these models has been the structure of the nucleon itself.

Perhaps the most natural way to include the mesonic degrees of freedom in a constituent quark model study of the NN interaction is to incorporate quarkantiquark excitations directly into the model space. The basic quark-gluon interaction lagrangian includes $q \bar{q}$ pair creation terms which lead to off-shell matrix elements coupling the $3 q$ configurations of the nucleon to $3 q+q \bar{q}$ components. It is the purpose of this investigation to examine the consequences of such excitations for the NN interaction. The ultimate goal is to expand the RGM calculations for NN scattering to include three-cluster configurations of $3 q+3 q+q \bar{q}$ type and perhaps even more complicated $q \bar{q}$ excitations. Since the emphasis is on $q \bar{q}$ exchange a coordinate representation study in the framework of the resonating group method is particularly well suited for such a study. Since the full three-cluster treatment is extremely rich, a simpler approach is adopted in this first investigation. In the first step the $3 q$ component of the single nucleon is augmented by $3 q+q \bar{q}$ excitations. Even if the relative motion of the $3 q$ and $q \bar{q} 0$ s clusters is restricted to the energetically lowest p-wave the possible number of color, spin, and isospin combinations leads to 24 different $3 q+q \bar{q}$ states. In the next step, quark exchange kernels are calculated for the two-nucleon system descrihed hy these improved nucleon internal functions. Since the largest amplitudes of the $3 q+q \bar{q}$ components are of order $c \leqslant 0.25$ only terms of first order in these c's are retained in the calculation of the exchange 
kernels. Finally, these exchange kernels are converted to phase-shift-equivalent local potentials by the Wigner-transform WKB technique used in refs. ${ }^{32,33,13,16}$ ).

Sect. 2 gives the quark interaction with emphasis on the $q \bar{q}$ pair creation terms. The full formulation of the model is given in sect. 3 . The parameters of the model are still $\alpha_{\mathrm{s}}, m, b$ and $a_{\mathrm{c}}$, as in the simple $3 \mathrm{q}+3 \mathrm{q}$ model. Since cross-terms involving $3 q$ and $3 q+q \bar{q}$ components of the nucleon internal functions make significant contributions to the $\mathrm{N}$ - and $\Delta$-masses, the nucleon magnetic moments and rms radii the numerical values of these parameters must be reevaluated for the expanded model space. The selection of parameters is discussed in sect. 4. Since the $q \bar{q}$ pair-creation terms in the interaction can also be taken to determine the strengths of the nucleon-meson coupling constants, these lead to new constraints on the parameters. Since a simple q $\bar{q}$ cluster with pion quantum numbers cannot be expected to give a realistic picture of the pion we do not expect to fit the pion-nucleon coupling constant nor the extreme long-range OPEP part of the NN interaction. However, the model has been constrained to fit the vector-meson coupling constants. Moreover, it gives the observed tensor/vector coupling ratios for $\rho$ and $\omega$; and the vector-meson dominance of the electromagnetic form factors is naturally reproduced. Numerical results for S-wave-equivalent local potentials and phase shifts are shown in sect. 5. The most prominent results are: (i) the repulsive core heights predicted by the simple $3 q+3 q$ models are greatly reduced, (ii) the strong energy dependence of the respulsive cores survives, and (iii) the $q \bar{q}$ excitations lead to an attractive part in the equivalent potentials in the $0.8-1.5 \mathrm{fm}$ range. However, this attraction is too weak to bind the deuteron or reproduce the extreme low-energy S-wave phase shifts. A concluding section gives an analysis of these results with a discussion of the shortcomings of the model, pointing to future directions.

\section{The $q \bar{q} \bar{q}$ pair-creation interaction}

Previous quark-model studies have used the Fermi-Breit interaction (in dominant nonrelativistic approximation), based on the one-gluon exchange process. Since the basic quark-gluon interaction lagrangian includes $q \bar{q}$ creation and annihilation terms the full Breit interaction contains the five types of terms illustrated in fig. 1 . The quark-quark interaction ( $H_{\mathrm{qq}}$ in fig. 1) contains tensor and spin-orbit terms ${ }^{14,15}$ ).

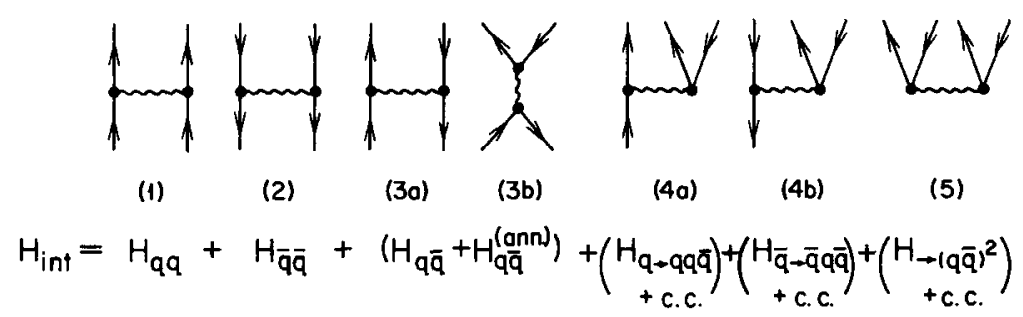

Fig. 1. The full Breit interaction hamiltonian. 
Since this investigation is restricted to $S$-wave scattering such terms can be omitted, and we use (with $H_{\mathrm{qq}}=\sum_{i<j} v_{i j}$ )

$$
v_{i j}=\alpha_{\mathrm{s}} \hbar c \frac{1}{4}\left(\lambda_{i} \cdot \lambda_{j}\right)\left\{\frac{1}{r_{i j}}-\frac{\pi \hbar^{2}}{m^{2} c^{2}} \delta\left(\boldsymbol{r}_{i j}\right)\left[1+\frac{2}{3}\left(\boldsymbol{\sigma}_{i} \cdot \boldsymbol{\sigma}_{j}\right)\right]\right\}-\left(\lambda_{i} \cdot \lambda_{j}\right) a_{\mathrm{c}} r_{i j}^{2},
$$

where a quadratic confining potential has been added to the gluon exchange terms. The SU(3) color generators have the standard normalization, $\left(\lambda_{i} \cdot \lambda_{i}\right)=\frac{16}{3}$. The $\overline{\mathrm{q}} \overline{\mathrm{q}}$ and $q \bar{q}$ interaction, diagrams (2) and (3a) lead to a potential of the same form, the latter because the minus sign in $H_{\mathrm{q} \overline{\mathrm{q}}}=-H_{\mathrm{qq}}$ is balanced by another change of sign in the $\mathrm{SU}(3)$ reduced matrix element which follows from $\mathrm{SU}(3)$ conjugation under the $\mathrm{q} \rightarrow \overline{\mathrm{q}}$ transformation. Due to the approximations inherent in (1), $\alpha_{\mathrm{s}}$ and $m$ are considered as parameters of the model. The central part of the momentum-dependent Breit retardation (or Darwin-like) term has been omitted in (1), despite the fact that it may be significant in lowering the repulsive core ${ }^{16}$ ), mainly for simplicity. The emphasis of this investigation is on the additional effects of q $\bar{q}$ excitations.

The annihilation diagram contribution to the $q \bar{q}$ interaction, ( $3 b)$, has been used by Faessler $e t$ al. ${ }^{34}$ ) in a study of the proton-antiproton interaction. It leads to

$$
v_{i j}^{(\mathrm{ann})}=\alpha_{\mathrm{s}} \frac{\pi}{6} \frac{\hbar^{3}}{m^{2} c} \delta\left(\boldsymbol{r}_{i j}\right) \frac{\left(1-\tau_{i} \cdot \tau_{j}\right)}{2}\left(\frac{\boldsymbol{\sigma}_{i}+\boldsymbol{\sigma}_{j}}{2}\right)^{2}\left(\frac{\lambda_{i}+\lambda_{j}}{2}\right)^{2} .
$$

Diagrams (4) and (5) have no on-shell matrix elements. However, their off-shell contributions are relevant for the nucleon system with dominant $3 q$ component, for which diagram (4a) is of greatest importance. Such $q \bar{q}$ creation terms in the interaction follow from time-dependent perturbation theory and have recently been discussed in conncction with nuclcon-meson coupling constants by $\mathrm{Yu}$ and Zhang ${ }^{35}$ ). They can be put in the form

$$
H_{\mathrm{q} \rightarrow \mathrm{qqq}}=\sum_{\alpha \beta \gamma \delta}\left\langle\psi_{\gamma}(1) \psi_{\delta}(2)\left|V_{\mathrm{q} \rightarrow \mathrm{qq} \bar{q}}(1,2)\right| \psi_{\alpha}(1) \psi_{\bar{\beta}}(2)\right\rangle a_{\gamma}^{\dagger} a_{\delta}^{\dagger} b_{\beta}^{\dagger} a_{\alpha}
$$

with

$$
\begin{aligned}
V_{\mathrm{q} \rightarrow \mathrm{qq} \overline{\mathrm{q}}}(1,2) & =\frac{i}{2 \pi} \alpha_{\mathrm{s}} \hbar c \frac{1}{4}\left(\lambda_{1} \cdot \lambda_{2}\right) \sum_{j=1}^{2}\left(F^{(j)}\left(x_{1}, \boldsymbol{x}_{2}\right) \cdot \mathbf{0}^{(j)}(1,2)\right) . \\
\mathbf{0}^{(1)}(1,2) & =2 \boldsymbol{\sigma}_{2}-i\left[\boldsymbol{\sigma}_{1} \times \boldsymbol{\sigma}_{2}\right], \quad \mathbf{0}^{(2)}(1,2)=2 \boldsymbol{\sigma}_{2} \\
\boldsymbol{F}^{(1)}\left(\boldsymbol{x}_{1}, \boldsymbol{x}_{2}\right) & =\frac{\partial}{\partial r} F(r), \quad \boldsymbol{F}^{(2)}\left(x_{1}, \boldsymbol{x}_{2}\right)=i F(r) \boldsymbol{p}_{1}^{\mathrm{op}}
\end{aligned}
$$

where

$$
\begin{gathered}
F(r)=\frac{\pi}{2} \frac{\hbar}{m c} \frac{1}{r} \\
r=x_{1}-x_{2}, \quad p_{1}^{\mathrm{op}}=\frac{1}{i} \frac{\partial}{\partial x_{1}} .
\end{gathered}
$$


Following the philosophy of eq. (1) only the dominant term in the $\hbar / m c$ expansion has been retained in eq. (2e). The effect of a second-order term, $-i(\hbar / m c)^{2}\left(\boldsymbol{r} \cdot p_{1}^{\text {op }}\right) / r^{2}$, in $F(r)$ has been considered $\left.{ }^{35}\right)$. Since it does not lead to major changes this term has been omitted in the numerical calculations. To be consistent with eq. (1), we let the parameters $\alpha_{\mathrm{s}}$ and $m$ carry the effects of "higher order" terms. In eq. (2) the $\psi_{\alpha}$ are nonrelativistic space-spin, isospin, color wave functions,

$$
\psi_{\alpha}(1)=\mathrm{e}^{i\left(p_{\alpha} \cdot x_{1}\right)} w_{m_{s \alpha}} v_{m_{t \alpha}} c_{(10) \nu_{\alpha}},
$$

with plane-wave spatial part (unit-volume box normalization), and

$$
\psi_{\bar{\beta}}(2)=\mathrm{e}^{-i\left(\boldsymbol{p}_{\beta} \cdot \boldsymbol{x}_{2}\right)}(-1)^{\frac{1}{2}+m_{s \beta}} \boldsymbol{W}_{-m_{s \beta}}(-1)^{\frac{1}{1}+m_{t \beta}} v_{-m_{i \beta}}(-1)^{\phi\left(\nu_{\beta}\right)} \boldsymbol{c}_{(10) \nu_{\beta}} .
$$

The Elliott SU(3) notation is used for the color degree of freedom. The color triplet has $(\lambda \mu)=(10)$; and the color phase factor $\phi(\nu)$ is defined by the $3 \times \overline{3} \rightarrow 1 \mathrm{SU}(3)$ Wigner coefficient

$$
\sqrt{3}\left\langle(10) \nu ;(01) \nu^{c} \mid(00)\right\rangle=(-1)^{\phi(\nu)} .
$$

By carrying out the spin, isospin and color parts of the matrix elements, eq. (2) can be put in the form

$$
\begin{aligned}
& H_{\mathrm{q} \rightarrow \mathrm{qq \overline {q }}}=\frac{i}{2 \pi} \alpha_{\mathrm{s}} \hbar c 8 \sqrt{\frac{1}{3}} \sum_{\boldsymbol{S}_{\mathrm{f}} \boldsymbol{p}_{\gamma} \boldsymbol{p}_{\delta} \boldsymbol{p}_{\alpha} \boldsymbol{p}_{\beta}}\left\{3 \delta_{S_{\mathrm{f}}^{3}}\left\langle\boldsymbol{p}_{\gamma}, \boldsymbol{p}_{\delta}\left|\boldsymbol{F}^{(1)}\right| \boldsymbol{p}_{\alpha},-\boldsymbol{p}_{\beta}\right\rangle\right. \\
& \left.+\left(2 \delta_{S_{t_{2}^{3}}}+\sqrt{2} \delta_{S_{\left[\frac{1}{2}\right.}}\right)\left\langle\boldsymbol{p}_{\gamma}, \boldsymbol{p}_{\delta}\left|\boldsymbol{F}^{(2)}\right| \boldsymbol{p}_{\alpha},-\boldsymbol{p}_{\beta}\right\rangle\right\} \\
& \times\left[\left[a_{p_{\gamma}}^{+\frac{11}{2}(10)} \times\left[a_{p_{\delta}}^{+\frac{1}{2} \frac{1}{2}(10)} \times b_{p_{\beta}}^{+\frac{1}{2}(01)}\right]^{10(11)}\right]^{S_{\mathrm{f}}^{\frac{1}{2}(10)}} \times a_{p_{\alpha}}^{\frac{11}{2}(01)}\right]^{10(00)},
\end{aligned}
$$

where the square brackets denote spin, isospin, and color coupling. In this form it can be seen directly that the additional $q \bar{q}$ pair which is created by the interaction has the gluon quantum numbers $S=1, T=0$, and $(\lambda \mu)=(11)$, i.e. color octet character. This spin, isospin, color character has the immediate consequence that the coupling matrix element between the $3 q$ component and the $3 q+q \bar{q}$ component has a direct term only if the final state has color octet $3 q$ and $q \bar{q}$ configurations. The spatial part of the interaction is made up of two different types of vector functions. The first, with index $j=1$, involves the gradient of the scalar relative coordinate function $F(r)$. It will also be denoted as D ("derivative") type, and gives non-zero matrix elements only when the final state of two quarks and one antiquark is coupled to the resultant spin $S_{\mathrm{f}}=\frac{3}{2}$. The second, with $j=2$, which has the extra momentum operator $\boldsymbol{p}_{1}^{\text {op }}$, and is therefore also denoted as M ("momentum") type, has non-zero matrix elements for both $S_{\mathrm{f}}=\frac{1}{2}$ and $\frac{3}{2}$. It should be noted that eq. (5) involves a scalar product of the spatial vector function matrix elements with the full quark-operator function which has vector character in spin space, $S-1$. Finally, in plane-wave representation the spatial integrals in eq. (5) will contain the momentum conservation factor $\delta_{\boldsymbol{p}_{\alpha},\left(\boldsymbol{p}_{\gamma}+\boldsymbol{p}_{\delta}+p_{\beta}\right)}$. 
Since the RGM formulation will require explicit antisymmetrization of identical particles located in different clusters, and since the final nucleon wave functions have components with different numbers of particles, it will also be useful to express the pair-creation interaction of eq. (2) in full coordinate representation. Quarks and antiquarks will be considered as distinguishable. If $x_{i}$ stands for a complete set of spin, isospin, and color internal as well as space coordinates $\left(\boldsymbol{x}_{i}\right)$, the full coordinate representation amplitude of a system of $A$ quarks and $B$ antiquarks is given by

$$
\begin{aligned}
\left\langle x_{1} \cdots\right. & \left.x_{A} ; x_{A+1} \cdots x_{A+B}\left|b^{\dagger}\left(x_{A+B}^{\prime}\right) \cdots b^{+}\left(x_{A+1}^{\prime}\right) a^{\dagger}\left(x_{A}^{\prime}\right) \cdots a^{\dagger}\left(x_{1}^{\prime}\right)\right| 0\right\rangle \\
= & \frac{1}{\sqrt{A !}} \sum_{P \in S_{A}} \delta_{P} P\left\{\delta\left(x_{1}-x_{1}^{\prime}\right) \cdots \delta\left(x_{A}-x_{A}^{\prime}\right)\right\} \\
& \times \frac{1}{\sqrt{B !}} \sum_{Q \in S_{B}} \delta_{Q} Q\left\{\delta\left\{x_{A+1}-x_{A+1}^{\prime}\right) \cdots \delta\left(x_{A+B}-x_{A+B}^{\prime}\right)\right\} .
\end{aligned}
$$

The state

$$
\begin{aligned}
\left|f_{A B}\right\rangle= & \int \mathrm{d} x_{1}^{\prime} \cdots \mathrm{d} x_{A+B}^{\prime} \hat{f}_{A B}\left(x_{1}^{\prime}, \ldots, x_{A+B}^{\prime}\right) b^{\dagger}\left(x_{A+B}^{\prime}\right) \\
& \cdots b^{\dagger}\left(x_{A+1}^{\prime}\right) a^{\dagger}\left(x_{A}^{\prime}\right) \cdots a^{\dagger}\left(x_{1}^{\prime}\right)|0\rangle
\end{aligned}
$$

is represented by the $(A+B)$-particle coordinate wave function

$$
\begin{aligned}
\left\langle x_{1} \cdots x_{A+B} \mid f_{A B}\right\rangle & \equiv f_{A B}\left(x_{1}, \ldots, x_{A+B}\right) \\
& =\frac{1}{\sqrt{A !}} \sum_{P \in S_{A}} \delta_{P} P \frac{1}{\sqrt{B !}} \sum_{Q \in S_{B}} \delta_{Q} Q \hat{f}_{A B}\left(x_{1}, \ldots, x_{A+B}\right) .
\end{aligned}
$$

In eqs. (6) the permutation operators $P$ and $Q$ act in the separate spaces of the two families of fermions, $A$ quarks and $B$ antiquarks (with $\delta_{P}= \pm 1$ for $P$ even/odd). In this representation the pair-creation interaction of eq. (2a) has the form

$$
H_{\mathrm{q} \rightarrow \mathrm{qq} \bar{q}}=\int \mathrm{d} x_{1} \mathrm{~d} x_{2} \mathrm{~d} x_{3} U_{\mathrm{q} \rightarrow \mathrm{qq} \overline{\mathrm{q}}}\left(x_{1} ; x_{2}, x_{3}\right) a^{\dagger}\left(x_{1}\right) a^{\dagger}\left(x_{2}\right) b^{\dagger}\left(x_{3}\right) a\left(x_{1}\right),
$$

where $U_{\mathrm{q} \rightarrow \mathrm{qq} \bar{q}}$ follows from the $V_{\mathrm{q} \rightarrow \mathrm{qq} \bar{q}}$ of eqs. (2b)-(2f) through

$$
\begin{aligned}
& \left\langle\psi_{\gamma}(1) \psi_{\delta}(2)\left|V_{\mathrm{q} \rightarrow \mathrm{qq} \overline{\mathrm{q}}}(1,2)\right| \psi_{\alpha}(1) \psi_{\bar{\beta}}(2)\right\rangle \\
& \quad=\left\langle\psi_{\gamma}(1) \psi_{\delta}(2) \psi_{\beta^{-1}(3) \mid}\left|U_{\mathrm{q} \rightarrow \mathrm{qq} \overline{\mathrm{q}}}\left(x_{1} ; x_{2}, x_{3}\right)\right| \psi_{\alpha}(1)\right\rangle,
\end{aligned}
$$

with

$$
\psi_{\beta^{-1}}(3)=\mathrm{e}^{i\left(p_{\beta} \cdot x_{3}\right)} w_{m_{s \beta}} v_{m_{1 \beta}} c_{(01)_{\beta}^{c}} .
$$

The explicit expression for $V_{\mathrm{q} \rightarrow \mathrm{qq} \overline{\mathrm{q}}}$ is given by the spin, isospin, color operators $\mathscr{S}^{(j)}(1 ; 2 ; 3)$ of the pair-creation interaction as

$$
U_{\mathrm{q} \rightarrow \mathrm{q} q \bar{q}}\left(x_{1} ; x_{2}, x_{3}\right)=i \frac{2}{\pi} \alpha_{\mathrm{s}} \hbar c \delta\left(x_{3}-x_{2}\right) \sum_{j=1}^{2}\left(F^{(j)}\left(x_{1}, x_{2}\right) \cdot \mathscr{P}^{(j)}(1 ; 2,3)\right),
$$


where

$$
\begin{aligned}
& \mathscr{S}_{\mu}^{(1)}(1 ; 2,3)=\mathscr{P}_{\mu}^{(2)}(1 ; 2,3)-\left[\sigma(1) \lambda^{(11)}(1) \times V^{10(11)}(2,3)\right]_{\mu}^{10(00)}, \\
& \mathscr{P}_{\mu}^{(2)}(1 ; 2,3)=\sqrt{2}\left[\lambda^{(11)}(1) \times V^{10(11)}(2,3)\right]_{\mu}^{10(00)}, \\
& V_{\mu 0 \nu}^{10(11)}(2,3)=[w(2) \times w(3)]_{\mu}^{1}[v(2) \times v(3)]_{0}^{0}\left[c_{(10)}(2) \times c_{(01)}(3)\right]_{\nu}^{(11)},
\end{aligned}
$$

and $\lambda^{(11)}(i)$ are the tensor representations of the SU(3) color generators $\lambda_{i \cdot}$ The many-particle matrix element is then calculated by the simple formula

$$
\begin{aligned}
\left\langle g_{A+1, B+1}\left|H_{\mathrm{q} \rightarrow \mathrm{qq} \overline{\mathrm{q}}}\right| f_{A B}\right\rangle & \\
= & (-1)^{B+1}[(A+1)(B+1)]^{1 / 2} \int \mathrm{d} x_{1} \cdots \mathrm{d} x_{A+B+2} g_{A+1, B+1}^{*}\left(x_{1}, \ldots, x_{A+B+2}\right) \\
& \times \sum_{i=1}^{A} U_{\mathrm{q} \rightarrow \mathrm{qq} \overline{\mathrm{q}}}\left(x_{i} ; x_{A+B+1}, x_{A+B+2}\right) f_{A B}\left(x_{1}, \ldots, x_{A+B}\right) .
\end{aligned}
$$

\section{Formulation of the model}

As a first step in the process of building quark-antiquark excitations into our quark model of NN scattering, an improved single-nucleon wave function is constructed in which the $3 q$ component is augmented by the $(3 q)(q \bar{q})$ excitations generated by the pair-creation interaction. Since the full two-nucleon problem will be formulated in terms of the scattering of nucleons with momenta $\boldsymbol{K}$ and $-\boldsymbol{K}$, the single-nucleon wave function of good momentum $\boldsymbol{K}$ is constructed by means of the projection operator $P_{K}$,

$$
\boldsymbol{P}_{\boldsymbol{K}} \psi_{\mathrm{N}}=c_{0}(\boldsymbol{K}) \mathrm{e}^{i \boldsymbol{K} \cdot \boldsymbol{X}_{3}} \phi_{0}(3 q)+\sum_{\alpha \neq 0}^{24} c_{\alpha}(\boldsymbol{K}) \mathrm{e}^{i \boldsymbol{K} \cdot \boldsymbol{X}_{\mathrm{s}}} \phi_{\alpha}((3 \mathrm{q})(\mathrm{q} \overline{\mathrm{q}})),
$$

where the coordinates of the center of mass

$$
X_{3}=\frac{1}{3}\left(x_{1}+x_{2}+x_{3}\right), \quad X_{5}=\frac{1}{5}\left(x_{1}+x_{2}+x_{3}+x_{4}+x_{5}\right)
$$

must be expressed in different form for the two types of components of $\psi_{\mathrm{N}}$ since these carry different mass numbers.

The dominant $3 \mathrm{q}$ component is given in terms of the usual internal wave function $\phi_{0}(3 q)$, with [3] symmetry for both space and spin-isospin functions, and [ $\left.1^{3}\right]$ color singlet, $(\lambda \mu)=(00)$ character, and 0 s harmonic oscillator internal relative motion functions. The $(3 q)(q \tilde{q})$ components are given in terms of two-cluster RGM wave functions

$$
\phi_{\alpha}((3 \mathrm{q})(\mathrm{q} \overline{\mathrm{q}}))=i N_{\alpha \alpha}^{-1 / 2} \mathscr{A}^{\prime}\left[\chi_{0 \mathrm{p}}^{\mathrm{h} . \mathrm{o}}(\boldsymbol{r})\left[\phi_{S_{1} T_{1}(\lambda \lambda)}(3 \mathrm{q}) \phi_{S_{2} T_{2}(\lambda \lambda)}(\mathrm{q} \overline{\mathrm{q}})\right]_{S_{12} T(00)}\right]_{S M_{S}},
$$

which are antisymmetrized explicitly with respect to the four quarks by

$$
\mathscr{A}^{\prime}=\sqrt{\frac{1}{4}}\left(1-P_{14}-P_{24}-P_{34}\right) \text {. }
$$

The factor $i$ in eq. (12) has been introduced to yield real values for the $c_{\alpha}$. The vector character of the pair-creation interaction automatically induces the required 
p-wave character of the function $\chi(r)$ describing the relative motion of the two 0 s clusters. For simplicity $\chi(r)$ is chosen to be a harmonic-oscillator function of $1 \hbar \omega$ excitation with the same oscillator length parameter $b$ used for the 0 s internal relative motion functions of the $3 q$ and $q \bar{q}$ clusters. The suffix $\alpha$ is used to designate the spin, isospin, color quantum numbers of the clusters. The index $\alpha=0$ is reserved for the pure (3q) component. Despite the requirement that the full $(3 q)(q \bar{q})$ system have color singlet character with the resultant $S T=\frac{11}{2} \frac{1}{2}$ of the nucleon, there are 24 possible values of $\alpha(\alpha=1, \ldots, 24)$, with $\alpha \equiv\left[S_{1} T_{1}(\lambda \lambda), S_{2} T_{2}(\lambda \lambda)\right] S_{12}$. With $(\lambda \lambda)=$ (00) the possible $S_{1} T_{1}$ values are $\frac{1}{2} \frac{1}{2}$ and $\frac{33}{2}$ corresponding to the case of the $\mathrm{N}$ or $\Delta$ of the simple $3 q$ model. There are nine possible states of this color singlet type with the $S_{2} T_{2}$ combinations carrying the quantum numbers of the $\eta, \pi, \omega$, and $\rho$-mesons, in some cases with $S_{12}=\frac{1}{2}$ and $\frac{3}{2}$. In addition there are 15 hidden color combinations with $(\lambda \lambda)=(11)$. In these both the $3 q$ spin-isospin and color functions have [21] symmetry, so that the possible $S_{1} T_{1}$ values are $\frac{11}{2}, \frac{31}{2}, \frac{1}{2} \frac{3}{2}$ to be designated $N^{c}$ and $C_{1}, C_{2}$, respectively; the notation $N^{c}$ indicates that the $3 q$ cluster carries the spin, isospin quantum members of the nucleon but is a color octet, similar designations, $\eta^{c}, \pi^{c}, \omega^{c}, \rho^{c}$, hold for the q $\bar{q}$ clusters. The full set of the 24 combinations of $\left[S_{1} T_{1}(\lambda \lambda) S_{2} T_{2}(\lambda \lambda)\right] S_{12}$ is enumerated in table 1. It should be noted that the $\phi_{\alpha}$ of eq. (12) are normalized $\left(\left\langle\phi_{\alpha} \mid \phi_{\alpha}\right\rangle=1\right)$, but in general the $\phi_{\alpha}$ are not orthogonal to each other,

$$
\left\langle\phi_{\alpha} \mid \phi_{\alpha^{\prime}}\right\rangle \neq 0 \quad \text { if } \alpha \neq \alpha^{\prime} .
$$

It is interesting to note that the $N_{\alpha \alpha}$ are all very close to 1 (see table 1), and the overlaps are all very close to zero. For example, the largest of all the $\left\langle\phi_{\alpha} \mid \phi_{\alpha^{\prime}}\right\rangle$ (with $\alpha=\Delta \pi, S_{12}=\frac{3}{2}$, and $\alpha^{\prime}=C_{1} \omega^{c}, S_{12}=\frac{3}{2}$ ) has the value 0.171 . Since the relative motion function in eq. (12) is chosen to be a $1 \hbar \omega$ excitation oscillator function, the $\phi_{\alpha}$ could have been expanded in terms of the $q^{4} \overline{\mathrm{q}}$ shell-model configurations $(0 s)^{3}(0 p)(\overline{0 s})$ and $(0 s)^{4}(\overline{0 p})$. There are altogether 30 shell-model components of this type with the appropriate total spin, isospin, color quantum numbers $\frac{1}{2} \frac{1}{2}(00)$. However, six combinations of these lead to spurious excitations of the center-of-mass motion. Even in harmonic-oscillator approximation, the removal of center-of-mass excitation is complicated. Moreover, it entails different approximations for the 3and 5-particle components of the nucleon wave functions. The states of eq. (10) which are momentum eigenstates of the nucleon, built with two-cluster RGM wave functions, are therefore to be preferred for the present study. However, the functions $\phi_{\alpha}$ have also been expanded in terms of such a $\mathrm{q}^{4} \overline{\mathrm{q}}$ shell-model basis to check the orders of magnitude of the resultant $c_{\alpha}$ against a single-nucleon calculation using zero-rest-mass MIT bag quark wave functions for the $q^{4} \bar{q}$ basis.

The evaluation of the $c_{\alpha}(K)$ follows from the variational principle

$$
\delta \frac{\left\langle\psi_{N}\left|H P_{K}\right| \psi_{N}\right\rangle}{\left\langle\psi_{N}\left|P_{K}\right| \psi_{N}\right\rangle}=0
$$


TABLE 1a

$(3 q)(q \bar{q})$ states with $(\lambda \lambda)=(00)$

\begin{tabular}{rccccccccr}
\hline$\alpha$ & Name & $S_{12}$ & $S_{1} T_{1} S_{2} T_{2}$ & $N_{\alpha \alpha}^{-1}$ & $X_{(34) 3}^{\mathrm{M}, \alpha}$ & $X_{(34) 2}^{\mathrm{D}, \alpha}$ & $X_{(34) 3}^{\mathrm{D}, \alpha}$ & $H_{\alpha 0}[\mathrm{MeV}]$ & \multicolumn{1}{c}{$c_{\alpha}$} \\
\hline 1 & $\mathrm{~N} \eta$ & $\frac{1}{2}$ & $\frac{1}{2} \frac{1}{2} 00$ & $\frac{24}{23}$ & $\frac{1}{9}$ & $-\frac{1}{18}$ & 0 & 234 & -0.057 \\
2 & $\mathrm{~N} \omega$ & $\frac{1}{2}$ & $\frac{1}{2} \frac{1}{2} 10$ & $\frac{72}{71}$ & $\frac{1}{9} \sqrt{\frac{1}{3}}$ & $\frac{1}{18} \sqrt{\frac{1}{3}}$ & $\frac{4}{9} \sqrt{\frac{1}{3}}$ & 133 & -0.056 \\
3 & $\mathrm{~N} \pi$ & $\frac{1}{2}$ & $\frac{1}{2} \frac{1}{2} 01$ & $\frac{72}{71}$ & $\frac{5}{9} \sqrt{\frac{1}{3}}$ & $-\frac{5}{18} \sqrt{\frac{1}{3}}$ & 0 & 665 & -0.182 \\
4 & $\mathrm{~N} \rho$ & $\frac{1}{2}$ & $\frac{1}{2} \frac{1}{2} 11$ & $\frac{216}{199}$ & $-\frac{7}{27}$ & $\frac{5}{54}$ & $-\frac{4}{27}$ & -556 & 0.135 \\
5 & $\Delta \rho$ & $\frac{1}{2}$ & $\frac{3}{2} \frac{3}{2} 11$ & $\frac{54}{53}$ & $\frac{4}{27} \sqrt{2}$ & $-\frac{1}{27} \sqrt{2}$ & $\frac{4}{27} \sqrt{2}$ & 436 & -0.108 \\
6 & $\mathrm{~N} \omega$ & $\frac{3}{2}$ & $\frac{1}{2} \frac{1}{2} 10$ & $\frac{18}{17}$ & $\frac{4}{9} \sqrt{\frac{1}{3}}$ & $-\frac{7}{36} \sqrt{\frac{1}{3}}$ & $\frac{7}{9} \sqrt{\frac{1}{3}}$ & 870 & -0.232 \\
7 & $\mathrm{~N} \rho$ & $\frac{3}{2}$ & $\frac{1}{2} \frac{1}{2} 11$ & $\frac{54}{55}$ & $\frac{8}{27}$ & $-\frac{17}{108}$ & $\frac{11}{27}$ & 967 & -0.255 \\
8 & $\Delta \pi$ & $\frac{3}{2}$ & $\frac{3}{2} \frac{3}{2} 01$ & $\frac{36}{37}$ & $-\frac{4}{9} \sqrt{\frac{1}{3}}$ & $\frac{7}{18} \sqrt{\frac{1}{3}}$ & 0 & -834 & 0.204 \\
9 & $\Delta \rho$ & $\frac{3}{2}$ & $\frac{3}{2} \frac{3}{2} 11$ & $\frac{108}{109}$ & $\frac{4}{27} \sqrt{5}$ & $-\frac{5}{54} \sqrt{5}$ & $\frac{4}{27} \sqrt{5}$ & 1086 & -0.257 \\
\hline
\end{tabular}

TABLE 1 b

Hidden color states with $(\lambda \lambda)=(11)$

\begin{tabular}{rcccccccrr}
\hline$\alpha$ & Name & $S_{12}$ & $S_{1} T_{1} S_{2} T_{2}$ & $N_{\alpha \alpha}^{-1}$ & $X_{(34) 3}^{\mathrm{M}, \alpha}$ & $X_{(34) 2}^{\mathrm{D}, \alpha}$ & $X_{(34) 3}^{\mathrm{D}, \alpha}$ & $H_{\alpha 0}[\mathrm{MeV}]$ & \multicolumn{1}{c}{$c_{\alpha}$} \\
\hline 10 & $\mathrm{~N}^{c} \eta^{c}$ & $\frac{1}{2}$ & $\frac{1}{2} \frac{1}{2} 00$ & $\frac{12}{13}$ & $\frac{1}{18}$ & $-\frac{1}{36}$ & 0 & 110 & -0.022 \\
11 & $\mathrm{~N}^{c} \omega^{c}$ & $\frac{1}{2}$ & $\frac{1}{2} \frac{1}{2} 10$ & $\frac{36}{37}$ & $-\frac{1}{9} \sqrt{\frac{1}{3}}$ & $\frac{1}{36} \sqrt{\frac{1}{3}}$ & $-\frac{1}{9} \sqrt{\frac{1}{3}}$ & 278 & -0.047 \\
12 & $\mathrm{~N}^{c} \pi^{c}$ & $\frac{1}{2}$ & $\frac{1}{2} \frac{1}{2} 01$ & $\frac{36}{37}$ & $\frac{1}{9} \sqrt{\frac{1}{3}}$ & $\frac{7}{36} \sqrt{\frac{1}{3}}$ & 0 & 28 & -0.016 \\
13 & $\mathrm{~N}^{c} \rho^{c}$ & $\frac{1}{2}$ & $\frac{1}{2} \frac{1}{2} 11$ & $\frac{108}{101}$ & $-\frac{1}{54}$ & $-\frac{7}{108}$ & $\frac{1}{27}$ & 22 & 0.005 \\
14 & $C_{1} \omega^{c}$ & $\frac{1}{2}$ & $\frac{3}{2} \frac{1}{2} 10$ & $\frac{18}{17}$ & $\frac{1}{18} \sqrt{\frac{1}{3}}$ & $-\frac{5}{36} \sqrt{\frac{1}{3}}$ & $\frac{1}{18} \sqrt{\frac{1}{3}}$ & -92 & 0.021 \\
15 & $C_{1} \rho^{c}$ & $\frac{1}{2}$ & $\frac{3}{2} \frac{1}{2} 11$ & $\frac{54}{55}$ & $-\frac{1}{54}$ & $-\frac{13}{108}$ & $-\frac{1}{54}$ & 51 & 0.003 \\
16 & $C_{2} \pi^{c}$ & $\frac{1}{2}$ & $\frac{1}{2} \frac{3}{2} 01$ & $\frac{18}{17}$ & $-\frac{1}{18} \sqrt{\frac{1}{3}}$ & $-\frac{2}{3} \sqrt{\frac{1}{3}}$ & 0 & 38 & 0.005 \\
17 & $C_{2} \rho^{c}$ & $\frac{1}{2}$ & $\frac{1}{2} \frac{3}{2} 11$ & $\frac{54}{55}$ & $\frac{5}{54}$ & $\frac{2}{27}$ & $\frac{4}{27}$ & 130 & -0.033 \\
18 & $\mathrm{~N}^{c} \omega^{c}$ & $\frac{3}{2}$ & $\frac{1}{2} \frac{1}{2} 10$ & $\frac{9}{10}$ & $\frac{1}{18} \sqrt{\frac{1}{3}}$ & $\frac{11}{72} \sqrt{\frac{1}{3}}$ & $\frac{1}{18} \sqrt{\frac{1}{3}}$ & -281 & 0.046 \\
19 & $\mathrm{~N}^{c} \rho^{c}$ & $\frac{3}{2}$ & $\frac{1}{2} \frac{1}{2} 11$ & $\frac{27}{29}$ & $\frac{5}{54}$ & $\frac{13}{216}$ & $\frac{4}{27}$ & 192 & -0.051 \\
20 & $C_{1} \eta^{c}$ & $\frac{3}{2}$ & $\frac{3}{2} \frac{1}{2} 00$ & $\frac{12}{13}$ & $-\frac{1}{18} \sqrt{\frac{1}{2}}$ & $\frac{1}{9} \sqrt{\frac{1}{2}}$ & 0 & -167 & 0.025 \\
21 & $C_{1} \omega^{c}$ & $\frac{3}{2}$ & $\frac{3}{2} \frac{1}{2} 10$ & $\frac{36}{37}$ & $\frac{1}{18} \sqrt{\frac{5}{6}}$ & $\frac{1}{36} \sqrt{\frac{5}{6}}$ & $\frac{1}{18} \sqrt{\frac{5}{6}}$ & -348 & 0.058 \\
22 & $C_{1} \pi^{c}$ & $\frac{3}{2}$ & $\frac{3}{2} \frac{1}{2} 01$ & $\frac{36}{35}$ & $\frac{1}{18} \sqrt{\frac{1}{6}}$ & $\frac{5}{36} \sqrt{\frac{1}{6}}$ & 0 & -3 & -0.010 \\
23 & $C_{1} \rho^{c}$ & $\frac{3}{2}$ & $\frac{3}{2} \frac{1}{2} 11$ & $\frac{108}{107}$ & $-\frac{1}{54} \sqrt{\frac{5}{2}}$ & $\frac{2}{27} \sqrt{\frac{5}{2}}$ & $-\frac{1}{54} \sqrt{\frac{5}{2}}$ & -197 & 0.024 \\
24 & $C_{2} \rho^{c}$ & $\frac{3}{2}$ & $\frac{1}{2} \frac{3}{2} 11$ & $\frac{54}{49}$ & $\frac{1}{27}$ & $\frac{2}{27}$ & $\frac{5}{54}$ & 39 & -0.012 \\
\hline
\end{tabular}

For states with $S_{2} T_{2}=10\left(\omega^{\mathrm{c}}\right), X_{e, 3}^{\mathrm{D}, \alpha}=\frac{2}{3} \sqrt{\frac{1}{3}}$ for $\left(S_{1} T_{1}\right) S_{12}=\left(\frac{1}{2} \frac{1}{2}\right) \frac{1}{2}, X_{e, 3}^{\mathrm{D}, \alpha}=-\frac{1}{3} \sqrt{\frac{1}{3}}$ for $\left(\frac{3}{2} \frac{1}{2}\right) \frac{1}{2}$ and $\left(\frac{1}{2} \frac{1}{2}\right) \frac{3}{2}$, and $X_{e, 3}^{\mathrm{D}, \alpha}=-\frac{1}{3} \sqrt{\frac{5}{6}}$ for $\left(\frac{3}{2} \frac{1}{2}\right) \frac{3}{2}$.

through the resultant secular equations

$$
\sum_{\alpha^{\prime}}\left\{H_{\alpha, \alpha^{\prime}}^{K}-E(K)\left\langle\phi_{\alpha} \mid \phi_{\alpha^{\prime}}\right\rangle\right\} c_{\alpha^{\prime}}(\boldsymbol{K})=0 .
$$

In eq. (15), $H$ represents the full hamiltonian which consists of the nonrelativistic kinetic energy part and $H_{\text {int }}$ of fig. 1. In principle, these should be solved for each $\boldsymbol{K}$, leading to $c_{\alpha}$ which are functions of $\boldsymbol{K}$. In practice, the equations are solved for 
$\boldsymbol{K}=0$, and since the interest is in low-energy NN scattering the $c_{\alpha}(\boldsymbol{K})$ are replaced with $K$-independent constants $c_{\alpha}=c_{\alpha}(0)$. It should also be noted that the plane-wave factors of eq. (10), with unit-volume box normalization, give center-of-mass integrals of unity when $K=0$.

The evaluation of the necessary interaction and norm kernels is carried out by standard techniques ${ }^{36-38}$ ). The $(3 q)$ and $(q \bar{q})$ internal energies are given (in terms of the parameters $\alpha_{\mathrm{s}}, m, b, a_{\mathrm{c}}$ and the combination $x=\hbar / m c b$ ) by

$$
\begin{aligned}
E_{S_{1} T_{1}(\lambda \lambda)}(3 q)= & \left(3+\frac{3}{2} x^{2}\right) m c^{2}+24 a_{\mathrm{c}} b^{2}+\sqrt{\frac{2}{\pi}} \alpha_{\mathrm{s}} x m c^{2}\left[-2+x^{2} \frac{2}{9} S_{1}\left(S_{1}+1\right)\right] \\
& +\delta_{(\lambda \lambda)(11)}\left\{-18 a_{\mathrm{c}} b^{2}+\sqrt{\frac{2}{\pi}} \alpha_{\mathrm{s}} x m c^{2}\left[\frac{3}{2}-\frac{1}{8} x^{2}\left(5 \delta_{S_{1} T_{1}, \frac{31}{2}}\right.\right.\right. \\
& \left.-3 \delta_{S_{1} T_{1,2 \frac{1}{2}}}+\delta_{\left.\left.S_{\mathrm{r}} T_{1,11} \frac{1}{2}\right)\right]}\right\} \\
E_{S_{2} T_{2}(\lambda \lambda)}(\mathrm{q} \overline{\mathrm{q}})= & \left(2+{ }_{4}^{3} x^{2}\right) m c^{2}+16 a_{\mathrm{c}} b^{2}+\sqrt{\frac{2}{\pi}} \alpha_{\mathrm{s}} x m c^{2}\left[-\frac{4}{3}+\frac{1}{3} x^{2}\left(\frac{4}{3} S_{2}\left(S_{2}+1\right)-1\right)\right] \\
& +\delta_{(\lambda \lambda)(11)}\left\{-18 a_{\mathrm{c}} b^{2}+\sqrt{\frac{2}{\pi}} \alpha_{\mathrm{s}} x m c^{2}\left[\frac{3}{2}-\frac{1}{8} x^{2}\left(5 \delta_{S_{2} T_{2}, 11}\right.\right.\right. \\
& \left.\left.\left.-3 \delta_{S_{2} T_{2}, 01}-3 \delta_{S_{2} T_{2}, 00}+\delta_{S_{2} T_{2}, 10}\right)\right]\right\}
\end{aligned}
$$

The interaction kernels lead to two types of matrix elements, the coupling matrix elements $H_{\alpha 0}$, connecting the $3 \mathrm{q}$ to the $(3 \mathrm{q})(\mathrm{q} \overline{\mathrm{q}})$ components, and the matrix elements $H_{\alpha \alpha^{\prime}}$ with both $\alpha, \alpha^{\prime} \neq 0$. The latter get contributions from the qq and qq interactions through the $v_{i j}$ of eq. (1) with $i<j(i, j=1, \ldots, 5)$, as well as the annihilation diagrams with $i=1, \ldots, 4$ and $j=5$. Both direct and exchange terms can make contributions. The different types of possible exchange terms are illustrated in fig. 2a for $H_{\mathrm{qq}}$ and $H_{\mathrm{q} \overline{\mathrm{q}}}$ and fig. $2 \mathrm{~b}$ for $H_{\mathrm{q} \overline{\mathrm{q}}}$ (annihilation). It is convenient to express the interaction matrix elements between the states of type $\phi_{\alpha}$ of eq. (12), with $1 \hbar \omega$ oscillator excitation, in terms of the simpler states of $0 \hbar \omega$ excitation (although the latter lead to negative-parity states of the baryon and are therefore not of interest in the present investigation). The results are $\left(\alpha, \alpha^{\prime} \neq 0\right)$

$$
\begin{gathered}
H_{\alpha \alpha^{\prime}}=N_{\alpha \alpha^{\prime}}^{-1 / 2} N_{\alpha^{\prime} \alpha^{\prime}}^{-1 / 2} \hat{H}_{\alpha \alpha^{\prime}}, \\
N_{\alpha \alpha^{\prime}}(0 \hbar \omega)=\delta_{\alpha \alpha^{\prime}}+X_{(34)-}^{1, \alpha \alpha^{\prime}}, \quad N_{\alpha \alpha^{\prime}}(1 \hbar \omega)=\delta_{\alpha \alpha^{\prime}}+\frac{1}{6} X_{(34)-}^{1, \alpha \alpha^{\prime}}, \\
\hat{H}_{\alpha \alpha^{\prime}}(0 \hbar \omega)=N_{\alpha \alpha^{\prime}}(0 \hbar \omega)\left(5+3 x^{2}\right) m c^{2}+\hat{H}_{\alpha \alpha^{\prime}}^{\mathrm{nn}}(0 \hbar \omega), \\
\hat{H}_{\alpha \alpha^{\prime}}(1 \hbar \omega)=N_{\alpha \alpha^{\prime}}(1 \hbar \omega)\left(5+\frac{7}{2} x^{2}\right) m c^{2}+\hat{H}_{\alpha \alpha^{\prime}}^{\mathrm{int}}(1 \hbar \omega),
\end{gathered}
$$




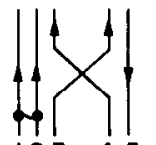

12345

$i j=12$

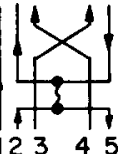

$i j=\quad \begin{array}{r}15 \\ 25\end{array}$

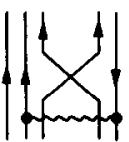

15
25

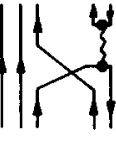

35

(b)

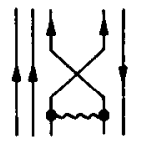

34

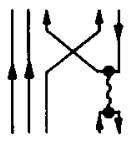

45
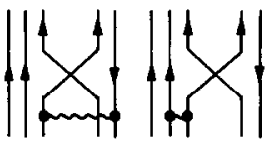

35

13
23

(a)

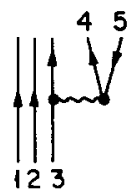

(Direct) $\quad \mathrm{i}=\begin{aligned} & 1 \\ & 2\end{aligned}$
45

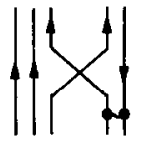

\section{(c)}

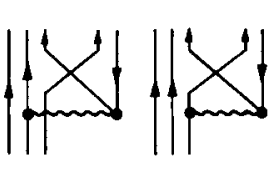

Fig. 2. The different types of quark-gluon exchange terms. (a) Exchange terms for $H_{\mathrm{qq}}$ and $H_{\mathrm{q} \overline{\mathrm{q}}}$ (b) Exchange terms for $H_{\mathrm{q} \bar{q}}$ (annihilation). (c) Direct and exchange terms for $H_{\mathrm{q} \rightarrow \mathrm{qq} \bar{q}}$.

$$
\begin{aligned}
& \hat{H}_{\alpha \alpha^{\prime}}^{\mathrm{int}}(0 \hbar \omega)=\left(-3 a_{\mathrm{c}} b^{2}+\right.\left.\frac{1}{4} \sqrt{\frac{2}{\pi}} \alpha_{\mathrm{s}} x m c^{2}\right)\left(X_{e}^{\mathrm{C}, \alpha \alpha^{\prime}}+X_{(34)}^{\mathrm{C}, \alpha \alpha^{\prime}}\right) \\
&+\sqrt{\frac{2}{\pi}} \alpha_{\mathrm{s}} x^{3} m c^{2}\left(X_{e}^{\mathrm{G}, \alpha \alpha^{\prime}}+X_{(34)}^{\mathrm{G}, \alpha \alpha^{\prime}}\right) \\
& \hat{H}_{\alpha \alpha^{\prime}}^{\mathrm{int}}(1 \hbar \omega)=\hat{H}_{\alpha \alpha^{\prime}}^{\mathrm{int}}(0 \hbar \omega)+\frac{5}{2} a_{\mathrm{c}} b^{2}\left(X_{(34)}^{\mathrm{C}, \alpha \alpha^{\prime}}-\frac{1}{3} \Delta X_{e}^{\mathrm{C}, \alpha \alpha^{\prime}}-\frac{1}{3} \Delta X_{(34)}^{\left.\mathrm{C}, \alpha \alpha^{\prime}\right)}\right) \\
&-\sqrt{\frac{2}{\pi}} \alpha_{\mathrm{s}} x m c^{2} \frac{5}{24}\left(X_{(34)}^{\mathrm{C}, \alpha \alpha^{\prime}}+\frac{1}{6} \Delta X_{e}^{\mathrm{C}, \alpha \alpha^{\prime}}+\frac{1}{6} \Delta X_{(34)}^{\mathrm{C}, \alpha \alpha^{\prime}}\right) \\
&-\sqrt{\frac{2}{\pi}} \alpha_{\mathrm{s}} x^{3} m c^{2} \frac{5}{6}\left(X_{(34)}^{\mathrm{G}, \alpha \alpha^{\prime}}+\frac{1}{2} \Delta X_{e}^{\mathrm{G}, \alpha \alpha^{\prime}}+\frac{1}{2} \Delta X_{(34)}^{\mathrm{G}, \alpha \alpha^{\prime}}\right)
\end{aligned}
$$

where

$$
X_{P}^{\mathrm{G}, \alpha \alpha^{\prime}}=-\frac{1}{16}\left(X_{P}^{\mathrm{C}, \alpha \alpha^{\prime}}+\frac{2}{3} X_{P}^{\mathrm{SC}, \alpha \alpha^{\prime}}\right)+\frac{1}{24} X_{P}^{\mathrm{ann}, \alpha \alpha^{\prime}} .
$$

In eqs. (19) $P$ indicates the quark permutation, with $P=1$ (designated by $e$ ) for the direct term, and $P=P_{34}$ (designated by (34)) for the exchange terms. The $X$-coefficients are given by the spin, isospin and color factors of the matrix elements of the spin, isospin, color-dependent operators $\mathcal{O}_{i j}$. If $\xi_{\alpha}$ is defined as the spin, isospin, color part of the function $\phi_{\alpha}$ then

$$
X_{P, i j}^{\sigma, u u^{\prime}}=\left\langle\xi_{\alpha}\left|\left\{\begin{array}{c}
\left(\lambda_{i} \cdot \lambda_{j}\right) \\
\left(\boldsymbol{\sigma}_{i} \cdot \boldsymbol{\sigma}_{j}\right)\left(\lambda_{i} \cdot \lambda_{j}\right) \\
\frac{\left(1-\tau_{i} \cdot \tau_{5}\right)}{2}\left(\frac{\sigma_{i}+\sigma_{5}}{2}\right)^{2}\left(\frac{\lambda_{i}+\lambda_{5}}{2}\right)^{2} \delta_{j, 5}
\end{array}\right\} C_{P} P\right| \xi_{\alpha^{\prime}}\right\rangle
$$


with

$$
C_{e}=1, \quad C_{(34)}=-3,
$$

for $O=C, S C$, and ann, respectively. For the exchange term of $N_{\alpha \alpha^{\prime}}$, with $\mathcal{O}=1$, $X_{(34)-}^{l, \alpha \alpha^{\prime}}$ designates the matrix element of $-3 P_{34}$ and the bar indicates that indices $i j$ are not required. In eq. (19) the $X$-coefficient without index $i j$ is defined by

$$
X_{P}^{O, \alpha \alpha^{\prime}}=\sum_{i<j} X_{P, i j}^{O, \alpha \alpha^{\prime}}
$$

Note that this sum involves seven distinct exchange terms since the spin, isospin, color matrix elements with $i j=15$ and 25 are equal; similarly for $i j=14,24$ and for $\ddot{j}=13,23$ (fig. 2a). For the annihilation diagram, with $j=5$ fixed, the sum over $i=1, \ldots, 4$ involves three distinct terms, shown in fig. $2 \mathrm{~b}$. The quantity $\Delta X_{\mathrm{p}}$ of eq. (19) is defined by

$$
\Delta X_{P}^{O, \alpha \alpha^{\prime}}=\sum_{i^{\prime} j^{\prime}} X_{P, i^{\prime} j^{\prime}}^{O, \alpha \alpha \alpha^{\prime}}-X_{P, i^{\prime \prime} j^{\prime \prime}}^{O, \alpha \alpha^{\prime}}
$$

where, for $P=1, i^{\prime} j^{\prime}=14,24,34,15,25,35$ and $i^{\prime \prime} j^{\prime \prime}$ are missing; and, for $P=P_{34}$, $i^{\prime} j^{\prime}=15,25, i^{\prime \prime} j^{\prime \prime}=34$ (but note that terms $i 4$ are missing in the annihilation diagrams). The $X_{P, i j}^{O}$ have been calculated with the c.f.p. expansions of the $3 \mathrm{q}$ states and the SU(2), SU(3) recoupling techniques illustrated in ref. ${ }^{39}$ ).

Finally, the coupling matrix elements, $H_{x \neq 0}$, connecting the $3 q$ to the $(3 q)(q \bar{q})$ components follow from eq. (9),

$$
H_{\alpha 0}=-\sqrt{4}\left\langle\phi_{\alpha}\left|\sum_{i=1}^{3} U_{\mathrm{q} \rightarrow \mathrm{qq} \hat{q}}\left(x_{i} ; x_{4}, x_{5}\right)\right| \phi_{0}\right\rangle \text {. }
$$

The $U_{q \rightarrow q q \bar{q}}$ involve two types of spatial vector functions, with $O=\mathrm{D}, \mathrm{M}$ in eq. (8), the D ("derivative") and $\mathrm{M}$ ("momentum") type; and the spin, isospin, color matrix elements of these will be designated by the superscripts $\sigma=\mathrm{D}$ and $\mathrm{M}$, respectively:

$$
X_{P, i}^{O, \alpha}=\left\langle P \xi_{\alpha}(1 \cdots 5)\left\|S^{\circ}(i ; 4,5)\right\| \xi_{0}(123)\right),
$$

where $\xi_{0}$ is the spin, isospin, color part of the function $\phi_{0}$. The reduced matrix elements in this paper are defined by: full matrix element $=$ (reduced matrix element) $\times($ Wigner coefficient $)$, without dimensional factors $\hat{j}=(2 J+1)^{1 / 2}$. The two types of exchange terms with $i=1$ or 2 , and $i=3$, are illustrated in fig. $2 \mathrm{c}$. Since the $\mathbf{M}$-type operator is independent of the spin operators associated with $x_{i}$ (see eq. $(2 c)$ ), its $x_{i}$ dependence is carried by the spatial variables $\left(x_{i}\right)$ and the color generator $\lambda_{i}$. Using the equality $X_{e, 1}^{\mathrm{M}, \alpha}=X_{e, 2}^{\mathrm{M}, \alpha}=X_{e, 3}^{\mathrm{M}, \alpha}$ it can be seen that the matrix elements of the direct term of M-type are proportional to the color matrix element of $\sum_{i=1}^{3} \lambda_{i *}$ Since this is the SU(3) color generator for the subspace of $\phi_{0}$, this SU(3) octet operator has zero matrix element even when connecting $\phi_{0}$ to a hidden color state in $\phi_{\alpha^{*}}$. Thus $\sum_{i} X_{e, i}^{M_{i} \alpha}=0$ for all $\alpha$, and there are no direct contributions to M-type matrix elements. Also, from $X_{\{34,1}^{M_{2} \alpha}=X_{(34), 2}^{M, \alpha}$ the same argument leads to 
$X_{(34), 2}^{\mathrm{M}, \alpha}=-\frac{1}{2} X_{(34), 3}^{\mathrm{M}, \alpha}$ for the exchange term of M-type. With these simplifications, the coupling matrix element $H_{\alpha 0}$ can be expressed as

$$
\begin{aligned}
H_{\alpha 0}= & N_{\alpha \alpha}^{-1 / 2} \alpha_{\mathrm{s}} \hbar c \frac{6}{\pi}\left(\hat{S}_{12} / \hat{S}\right)\left\{\left(I_{(34) 2}^{\mathrm{M}}-I_{(34) 3}^{\mathrm{M}}\right) X_{(34) 3}^{\mathrm{M}, \alpha}\right. \\
& \left.+I_{e, 3}^{\mathrm{D}} X_{e, 3}^{\mathrm{D}, \alpha}-2 I_{(34), 2}^{\mathrm{D}} X_{(34), 2}^{\mathrm{D}, \alpha}-I_{(34), 3}^{\mathrm{D}} X_{(34,3)}^{\mathrm{D}, \alpha}\right\} .
\end{aligned}
$$

The spatial integrals $I_{P, i}^{\circ}$ are standard reduced matrix elements for the two types of vector functions $\boldsymbol{F}^{O}$ (see eq. (2d)),

$$
I_{P, i}^{O}=\left\langle P \chi_{0 \mathrm{p}}^{\text {h.o. }}(r) \phi_{0 s}(3 \mathrm{q}) \phi_{0 \mathrm{~s}}(\mathrm{q} \tilde{\mathrm{q}})\left\|\delta\left(x_{4}-x_{5}\right) F^{O}\left(x_{i}, x_{4}\right)\right\| \phi_{0 \mathrm{~s}}(3 \mathrm{q})\right\rangle,
$$

where $P$ now stands for the space part of the permutation operator, and the $3 q$ and $q \bar{q}$ orbital functions involve the $0 \mathrm{~s}$ oscillator functions in the internal relative motion variables. With a $0 p$ oscillator function for the $(3 q)(q \bar{q})$ relative motion, this leads to

$$
\begin{aligned}
H_{\alpha 0}= & N_{\alpha \alpha}^{-1 / 2} \alpha_{\mathrm{s}} x^{2} m c^{2}\left(\frac{\sqrt{15}}{7}\right)^{3 / 2} \frac{2}{3}\left[\frac{5}{3 \pi}\right]^{1 / 2}\left(\hat{S}_{12} / \hat{S}\right) \\
& \times\left\{\frac{19}{2} X_{(34), 3}^{\mathrm{M}, \alpha}+6 X_{e, 3}^{\mathrm{D}, \alpha}-4 X_{(34), 2}^{\mathrm{D}, \alpha}+X_{(34), 3}^{\mathrm{D}, \alpha}\right\}
\end{aligned}
$$

The spin, isospin, color factors of the matrix elements are given in table 1. (Note that D-type matrix elements have a direct (e) contribution only for hidden color states with $(\mathrm{q} \overline{\mathrm{q}})$ quantum numbers of type $\omega^{c}$.) Table 1 also gives the numerical values of $H_{\alpha 0}$, as well as the final values of the $c_{\alpha}$, which are the key numbers for this investigation. Note that the $c_{\alpha}$ are normalized, according to

$$
c_{0}^{2}+\sum_{\alpha, \alpha^{\prime}=1}^{24} c_{\alpha} c_{\alpha^{\prime}}\left\langle\phi_{\alpha} \mid \phi_{\alpha^{\prime}}\right\rangle=1 .
$$

With the improved single-nucleon wave functions of eq. (10) the RGM equation for the NN relative motion function $\chi(R)$,

$$
\int \mathrm{d} \boldsymbol{R}^{\prime}\left[H\left(\boldsymbol{R}, \boldsymbol{R}^{\prime}\right)-E N\left(\boldsymbol{R}, \boldsymbol{R}^{\prime}\right)\right] \chi\left(\boldsymbol{R}^{\prime}\right)=0,
$$

is expressed in terms of kernels $H\left(\boldsymbol{R}, \boldsymbol{R}^{\prime}\right), N\left(\boldsymbol{R}, \boldsymbol{R}^{\prime}\right)$. For the $P_{\boldsymbol{K}} \psi_{\mathrm{N}}$ of eq. (10) with $\boldsymbol{K}$-dependent $c_{\alpha}(\boldsymbol{K})$, these would be generalizations of the standard RGM kernels involving the convolution functions

$$
\Delta_{\alpha \beta}\left(\boldsymbol{r}_{\alpha \beta}-\boldsymbol{R}\right)=\frac{1}{(2 \pi)^{3}} \int \mathrm{d} \boldsymbol{K} \mathrm{e}^{i \boldsymbol{K} \cdot\left(\boldsymbol{r}_{\alpha \beta}-\boldsymbol{R}\right)} c_{\alpha}(\boldsymbol{K}) c_{\beta}(-\boldsymbol{K})
$$

with

$$
\boldsymbol{H}\left(\boldsymbol{R}, \boldsymbol{R}^{\prime}\right)=\sum_{\alpha \beta \alpha^{\prime} \beta^{\prime}=0}^{24}\left\langle\mathscr{A}_{\alpha \beta} \Delta_{\alpha \beta}\left(\boldsymbol{r}_{\alpha \beta}-\boldsymbol{R}\right) \phi_{\alpha} \phi_{\beta}|H| \mathscr{A}_{\alpha^{\prime} \beta^{\prime}} \Delta_{\alpha^{\prime} \beta^{\prime}}\left(\boldsymbol{r}_{\alpha^{\prime} \beta^{\prime}}-\boldsymbol{R}^{\prime}\right) \phi_{\alpha^{\prime}} \phi_{\beta}\right\rangle .
$$


However, since the $c_{\alpha}(K)$ have been replaced by the $K$-independent $c_{\alpha}$, the $\Delta_{\alpha \beta}$ are replaced by $\delta$-functions,

$$
\Delta_{\alpha \beta}\left(\boldsymbol{r}_{\alpha \beta}-\boldsymbol{R}\right) \rightarrow c_{\alpha} c_{\beta} \delta\left(\boldsymbol{r}_{\alpha \beta}-\boldsymbol{R}\right),
$$

and the kernels go over to standard RGM kernels. The only direct terms arise from internal energy contributions and the kinetic energy part of $H$. (In general the direct term would get a contribution from the annihilation diagram. In the present investigation such terms which are at least of order $c_{\alpha}^{2}$ are neglected; see the discussion below.) With $E=E_{\text {internal }}+E_{\text {c.m. }}$, the RGM equation reduces to

$$
\left(E_{\mathrm{c} . \mathrm{m} .}-\frac{1}{M_{\mathrm{N}}} p_{\mathrm{op}}^{2}\right) \chi(\boldsymbol{R})=\int \mathrm{d} \boldsymbol{R}^{\prime} G\left(\boldsymbol{R}, \boldsymbol{R}^{\prime}\right) \chi\left(\boldsymbol{R}^{\prime}\right) .
$$

In principle the inertia parameter for the relative kinetic energy operator should be obtained from the solution of the $\boldsymbol{K}$-dependent single-nucleon problem. In practice, the experimental value of the two-nucleon reduced mass is used in the coefficient of the $p_{\mathrm{op}}^{2}$ term. However, the parameters $\alpha_{\mathrm{s}}, m, b$ and $a_{\mathrm{c}}$ are chosen such that $E_{\text {internal }}=2 M_{\mathrm{N}} c^{2}$, where $M_{\mathrm{N}}$ is the observed nucleon mass. The exchange kernel can be expanded in the $c_{\alpha}$ (with $\alpha \neq 0$ ),

$$
G\left(\boldsymbol{R}, \boldsymbol{R}^{\prime}\right)=c_{0}^{4} G_{0}\left(\boldsymbol{R}, \boldsymbol{R}^{\prime}\right)+\sum_{\alpha=1}^{24} c_{0}^{3} c_{\alpha} G_{\alpha}\left(\boldsymbol{R}, \boldsymbol{R}^{\prime}\right)+\cdots
$$

Since the $c_{\alpha} \leqslant 0.25$ (see table 1), terms of order $c_{\alpha}^{2}$ and higher will be neglected in constructing these kernels. In eq. (32), $G_{0}\left(R, R^{\prime}\right)$ designates the (3q)-(3q) part of the kernel, and $G_{\alpha}\left(\boldsymbol{R}, \boldsymbol{R}^{\prime}\right)$ is a cross term connecting the (3q)-(3q) part of the two-nucleon function to the $(3 q)-(3 q)(q \bar{q})_{\alpha}$ component.

It will be useful to replace $\chi(R)$ in the RGM equation with a function of good parity,

$$
\chi^{\mathscr{G}}(\boldsymbol{R})=\frac{1}{2}\left[\chi(\boldsymbol{R})-(-1)^{\mathscr{G}} \chi(-\boldsymbol{R})\right],
$$

with $\mathscr{S}=S+T$. Then

$$
G_{0}\left(\boldsymbol{R}, \boldsymbol{R}^{\prime}\right)=H^{(\mathrm{E})}\left(\boldsymbol{R}, \boldsymbol{R}^{\prime}\right)-\left(2 M_{\mathrm{N}} c^{2}+E_{\mathrm{c} . \mathrm{m} .}\right) N^{(\mathrm{E})}\left(\boldsymbol{R}, \boldsymbol{R}^{\prime}\right),
$$

where $H^{(\mathrm{E})}\left(\boldsymbol{R}, \boldsymbol{R}^{\prime}\right), N^{(\mathrm{E})}\left(\boldsymbol{R}, \boldsymbol{R}^{\prime}\right)$ are RGM exchange kernels for the simple (3q)-(3q) system with $\delta\left(\boldsymbol{r}_{00}-\boldsymbol{R}^{\prime}\right)$ replaced by

$$
\delta^{\mathscr{S}}\left(\boldsymbol{r}_{00}, \boldsymbol{R}^{\prime}\right)=\frac{1}{2}\left[\delta\left(\boldsymbol{r}_{00}-\boldsymbol{R}^{\prime}\right)-(-1)^{\mathscr{S}} \delta\left(\boldsymbol{r}_{00}+\boldsymbol{R}^{\prime}\right)\right] .
$$

It is useful to separate the fitted internal energy $2 M_{\mathrm{N}} c^{2}$ according to $2 M_{\mathrm{N}} c^{2}=$ $2 E_{0}(3 q)+2 \Delta M_{N} c^{2}$, where $E_{0}(3 q)$ is given by eq. (17) with $(\lambda \lambda)=(00)$. Also by using the symmetrized $\chi^{\mathscr{S}}(\boldsymbol{R})$ in the RGM equation (31), as well as parity conservation, $\boldsymbol{G}_{0}\left(\boldsymbol{K}, \boldsymbol{K}^{\prime}\right)$ can be expressed by

$$
G_{0}\left(\boldsymbol{R}, \boldsymbol{R}^{\prime}\right)=G_{00}\left(\boldsymbol{R}, \boldsymbol{R}^{\prime}\right)-\left(2 \Delta M_{\mathrm{N}} c^{2}+E_{\mathrm{c} . \mathrm{m} .}\right) N_{00}^{(\mathrm{E})}\left(\boldsymbol{R}, \boldsymbol{R}^{\prime}\right),
$$


where $G_{00}$ is the exchange kernel for the $3 q-3 q$ system,

$$
G_{00}\left(R, R^{\prime}\right)=-9\left(P_{36} \phi_{0} \phi_{0} \delta\left(r_{00}-R\right)\left|\left(H_{00}-2 E_{0}(3 q)\right)\right| \phi_{0} \phi_{0} \delta\left(r_{00}-R^{\prime}\right)\right\rangle,
$$

and

$$
N_{00}^{(\mathrm{E})}\left(\boldsymbol{R}, \boldsymbol{R}^{\prime}\right)=-9\left\langle\boldsymbol{P}_{36} \phi_{0} \phi_{0} \delta\left(\boldsymbol{r}_{00}-\boldsymbol{R}\right) \mid \phi_{0} \phi_{0} \delta\left(\boldsymbol{r}_{00}-\boldsymbol{R}^{\prime}\right)\right\rangle .
$$

These kernels are known in explicit analytic form, see e.g. ref. ${ }^{13}$ ). In eqs. (30) and (37), (and below), the spin, isospin coupling of $\phi_{0} \phi_{\beta}$ is, for simplicity of notation, not indicated explicitly.

The kernel $G_{\alpha}\left(R, R^{\prime}\right)$ in eq. (32) is related to the kernel of the pair creation

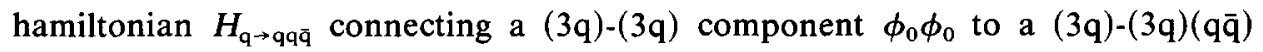
component $\phi_{0} \phi_{\alpha}$,

$$
G_{\alpha}\left(\boldsymbol{R}, \boldsymbol{R}^{\prime}\right)=H_{0 \alpha, 00}^{(\mathrm{E})}\left(\boldsymbol{R}, \boldsymbol{R}^{\prime}\right)+H_{0 \alpha, 00}^{(\mathrm{E})}\left(\boldsymbol{R}^{\prime}, \boldsymbol{R}\right)^{*} .
$$

Let us consider first the full RGM kernel given by eq. (9),

$$
\begin{aligned}
H_{0 \alpha, 00}\left(\boldsymbol{R}, \boldsymbol{R}^{\prime}\right)= & -\sqrt{7}\left\langle\mathscr{A}_{0 \alpha}^{\prime} \phi_{0} \phi_{\alpha} \delta\left(\boldsymbol{r}_{0 \alpha}-\boldsymbol{R}\right)\right| \\
& \times \sum_{i=1}^{6} U_{\mathrm{q} \rightarrow \mathrm{qq} \bar{q}}\left(x_{i} ; x_{7}, x_{8}\right)\left|\mathscr{A}_{00}^{\prime} \phi_{0} \phi_{0} \delta\left(\boldsymbol{r}_{00}-\boldsymbol{R}^{\prime}\right)\right\rangle,
\end{aligned}
$$

where, before antisymmetrization, the particles in the first $3 q$ cluster are labeled by $x_{1}, x_{2}, x_{3}$; in the second by $x_{4}, x_{5}, x_{6}$, and the $\mathrm{q} \bar{q}$ pair by $x_{7}, x_{8}$. The antisymmetrizer $\mathscr{A}_{00}^{\prime}$ is made up of a sum over the $\left(\begin{array}{c}6 \\ 3\end{array}\right)=20$ permutations which, besides the identity, include the permutations which exchange quarks between cluster $x_{1}, x_{2}, x_{3}$ and $x_{4}$, $x_{5}, x_{6}$; whereas $\mathscr{A}_{0 \alpha}^{\prime}$ is the similar sum of $\left(\begin{array}{l}7 \\ 3\end{array}\right)=35$ permutation operators which exchange quarks between the groups labeled $x_{1}, x_{2}, x_{3}$ and $x_{4}, x_{5}, x_{6}, x_{7}$. These have normalization factors of $\left(\begin{array}{l}6 \\ 3\end{array}\right)^{-1 / 2}$ and $\left(\begin{array}{l}7 \\ 3\end{array}\right)^{-1 / 2}$, respectively. Using the symmetry of $\sum_{i} U\left(x_{i} ; x_{7}, x_{8}\right), \mathscr{A}_{00}^{\prime}$ in eq. (39) can be simplified via

$$
-\sqrt{7} \mathscr{A}_{0 \alpha}^{\prime} \mathscr{A}_{00}^{\prime}=-\sqrt{7}\left(\begin{array}{l}
6 \\
3
\end{array}\right)^{1 / 2}\left(\begin{array}{l}
7 \\
3
\end{array}\right)^{-1 / 2} \sum_{P}^{\prime} \delta_{P} P=-\sqrt{4} \sum_{P}^{\prime} \delta_{P} P
$$

where the $\Sigma^{\prime}$ sum, 35 terms starting with the identity, contains in addition the permutations which exchange quarks between groups $x_{1}, x_{2}, x_{3}$ and $x_{4}, x_{5}, x_{6}, x_{7}$. Since $\phi_{\alpha}$ contains an additional antisymmetrizer

$$
\mathscr{A}_{\alpha}^{\prime}=\sqrt{\frac{1}{4}}\left(1-P_{47}-P_{57}-P_{67}\right),
$$

the additional identity

$$
-\sqrt{7} \mathscr{A}_{0 \alpha}^{\prime} \mathscr{A}_{00}^{\prime} \mathscr{A}_{\alpha}^{\prime}=-\sum_{P}^{\prime \prime} \delta_{P} P
$$


can be used. The $\Sigma^{\prime \prime}$ sum now contains $(7 ! / 3 ! 3 ! 1 !)=140$ terms which, besides the identity, includes all permutations which exchange quarks between the three clusters with labels $x_{1}, x_{2}, x_{3} ; x_{4}, x_{5}, x_{6}$; and $x_{7}$. Since the $\phi_{0}\left(x_{1}, x_{2}, x_{3}\right)$ and $\phi_{0}\left(x_{4}, x_{5}, x_{6}\right)$ are antisymmetrized, this sum can be replaced by an expansion over double coset generators ${ }^{40}$ ) for the $3+3+1$ three-cluster system,

$$
\begin{aligned}
\sum_{P}^{\prime \prime} \delta_{P} P= & \left(1-P_{0}\right)\left(1-3 P_{67}-3 P_{37}-9 P_{36}\right. \\
& \left.+9\left(P_{36} P_{37}+P_{37} P_{36}\right)+18\left(P_{36} P_{57}+P_{36} P_{27}\right)\right),
\end{aligned}
$$

where $P_{0} \equiv P_{14} P_{25} P_{36}$ exchanges all quarks between the two single-nucleon functions $\phi_{0}$. Since the exchange term in the RGM equation has the form

$$
\int G\left(\boldsymbol{R}, \boldsymbol{R}^{\prime}\right) \chi^{\mathscr{S}}\left(\boldsymbol{R}^{\prime}\right) \mathrm{d} \boldsymbol{R}^{\prime}=\int \frac{1}{2}\left[G\left(\boldsymbol{R}, \boldsymbol{R}^{\prime}\right)-(-1)^{\mathscr{S}} G\left(\boldsymbol{R},-\boldsymbol{R}^{\prime}\right)\right] \chi^{\mathscr{S}}\left(\boldsymbol{R}^{\prime}\right) \mathrm{d} \boldsymbol{R}^{\prime},
$$

and since

$$
\frac{1}{2}\left(1-P_{0}\right) \phi_{0} \phi_{0} \delta\left(r_{00}-R^{\prime}\right)=\phi_{0} \phi_{0} \delta^{\mathscr{S}}\left(r_{00}, R^{\prime}\right),
$$

the square bracket combination of eq. (44) is obtained if $\phi_{0} \phi_{0} \delta\left(r_{00}-\boldsymbol{R}^{\prime}\right)$ in eq. (39) is replaced by the left-hand side of (45). After hermitian conjugation of the product of antisymmetrizers in the bra side of $H_{0 \alpha, 00}$ the factor $\left(1-P_{0}\right)$ can be permuted to the right, and via $\left(1-P_{0}\right)^{2}=2\left(1-P_{0}\right)$, yields a factor 2 when acting on relation (45). Thus full antisymmetrization in the kernel of eq. (39) is accomplished by the eight terms of the right factor of eq. (43), if $\phi_{\alpha}$ is replaced by $\tilde{\phi}_{\alpha}$ where $\tilde{\phi}_{\alpha}$ is defined by

$$
\phi_{\alpha}=\mathscr{A}_{\alpha}^{\prime} \tilde{\phi}_{\alpha} .
$$

However, with this change, it must be remembered that the terms $1-3 P_{67}$ of eq. (43) contribute to the single-nucleon matrix elements and were part of the singlenucleon $H_{\alpha 0}$ which were used to calculate the single-nucleon energy. For the two-nucleon problem these are therefore part of $E_{\text {internal }}=2 M_{\mathrm{N}} c^{2}$ of eqs. (31)-(37) and must be omitted from the RGM kernel $H_{0 \alpha, 00}$. Finally, if $H_{0 \alpha, 00}$ is restored to the single $\delta$-function form of eq. (39) by using eqs. (44) and (45) again, then

$$
\begin{aligned}
H_{0 \alpha, 00}^{(\mathrm{E})}\left(\boldsymbol{R}, \boldsymbol{R}^{\prime}\right)= & -2 \sum_{P} C_{\boldsymbol{P}}\left\langle\boldsymbol{P} \phi_{0} \tilde{\phi}_{\alpha} \delta\left(\boldsymbol{r}_{0 \alpha}-\boldsymbol{R}\right)\right| \\
& \times \sum_{i=1}^{6} U_{\mathrm{q} \rightarrow q q \bar{q}}\left(x_{i} ; x_{7}, x_{8}\right)\left|\phi_{0} \phi_{0} \delta\left(\boldsymbol{r}_{00}-\boldsymbol{R}^{\prime}\right)\right\rangle,
\end{aligned}
$$

where

$$
\sum_{P} C_{P} P=-3 P_{37}-9 P_{36}+9 P_{36} P_{37}+9 P_{37} P_{36}+18 P_{36} P_{57}+18 P_{36} P_{77}
$$

Since the pair-creation interaction $U_{q \rightarrow q q a}$ involves a sum over the two types of 
terms $\mathrm{D}$ and $\mathrm{M}$, the exchange kernel can finally be expanded according to

$$
H_{0 \alpha, 00}^{(\mathrm{E})}\left(\boldsymbol{R}, \boldsymbol{R}^{\prime}\right)=\sum_{P} C_{P} \sum_{i=1}^{6} \sum_{O=\mathrm{D}, \mathrm{M}} G_{P, i}^{O, \alpha}\left(R, \boldsymbol{R}^{\prime}\right),
$$

where the notation parallels that of eqs. (24)-(26). The sums over $i$ and $P$ lead to 25 distinct types of exchange terms. For example, with $P=P_{37}$, terms with $i=4,5,6$ are identical, as are terms with $i=1,2$, while these are distinct from the term with $i=3$, so that $P_{37}$ leads to three distinct exchange terms. Similarly $P_{36}$, $P_{36} P_{37}, P_{37} P_{36}$ lead to four distinct terms each, while $P_{36} P_{57}, P_{36} P_{27}$ lead to five distinct exchange terms each. The $G_{P, i}^{O, \alpha}$ can again be factored into a spatial part and a spin, isospin, color factor $X_{P, i}^{O, \alpha}$ which is defined by

$$
\begin{aligned}
\left(X_{P, i}^{O_{,} \alpha}\right)^{S_{123}, S}= & \left\langle P\left[\xi_{0}(123) \times \xi_{\alpha}(4 \cdots 8)\right]_{S_{123} T(00)}\left\|\mathscr{S}^{O}(i ; 7,8)\right\|\right. \\
& \left.\times\left[\xi_{0}(123) \times \xi_{0}(456)\right]_{S T(00)}\right\rangle,
\end{aligned}
$$

where $\alpha(\neq 0)=\left[S_{1} T_{1}(\lambda \lambda), S_{2} T_{2}(\lambda \lambda)\right] S_{12}$.

For S-wave scattering the rearrangement of the orbital relative motion function in $\phi_{u}$ via an angular momentum transformation yields

$$
\begin{aligned}
G_{P, i}^{O, \alpha}\left(\boldsymbol{R}, \boldsymbol{R}^{\prime}\right)= & N_{\alpha \alpha}^{-1 / 2} \alpha_{\mathrm{s}} \hbar c \frac{8 \sqrt{3}}{\pi} \sum_{S_{123}} \frac{\left(\hat{S}_{123}\right)^{2}}{\hat{S}}\left\{\begin{array}{ccc}
0 & \frac{1}{2} & \frac{1}{2} \\
1 & S_{12} & \frac{1}{2} \\
1 & S_{123} & S
\end{array}\right\} \\
& \times I_{P, i}^{O}\left(\boldsymbol{R}, \boldsymbol{R}^{\prime}\right)\left(X_{P, i}^{O, \alpha}\right)^{S_{123}, S}
\end{aligned}
$$

where the spatial part of the kernel is given by

$$
\begin{aligned}
I_{P, i}^{\mathscr{O}}\left(\boldsymbol{R}, \boldsymbol{R}^{\prime}\right)= & \left\langle\boldsymbol{P} \phi_{0 \mathrm{~s}}(3 \mathrm{q}) \chi_{0 \mathrm{p}}^{\text {h.o. }}(\mathbf{p}) \phi_{0 \mathrm{~s}}(3 \mathrm{q}) \phi_{0 \mathrm{~s}}(\mathrm{q} \overline{\mathrm{q}}) \delta\left(\boldsymbol{r}_{0 \alpha}-\boldsymbol{R}\right) \|\right. \\
& \left.\times \delta\left(\boldsymbol{x}_{7}-\boldsymbol{x}_{8}\right) \boldsymbol{F}^{\mathcal{O}}\left(\boldsymbol{x}_{\mathrm{i}}, \boldsymbol{x}_{7}\right) \| \phi_{0 \mathrm{~s}}(3 \mathrm{q}) \phi_{0 \mathrm{~s}}(3 \mathrm{q}) \delta\left(\boldsymbol{r}_{00}-\boldsymbol{R}^{\prime}\right)\right\rangle .
\end{aligned}
$$

Note that in eq. (52) only the $l=0$ terms of the $\delta$-functions are assumed to contribute. The relative coordinate $\rho$ in $\phi_{\alpha}$ is defined as

$$
\rho=\frac{1}{2}\left(x_{7}+x_{8}\right)-\frac{1}{3}\left(x_{4}+x_{5}+x_{6}\right) \text {. }
$$

The spatial integrals in eq. (52) have been calculated by complex generator coordinate (Bargmann transform) techniques ${ }^{37.38}$ ). These techniques are particularly simple for operators with gaussian radial dependence so that functions such as $1 / r$ are expressed in terms of integrals over functions $\exp \left[-\chi^{2} r^{2}\right]$ and final evaluations are made numerically using 10-point Gauss-Legendre integration formulae.

To convert the kernels $G\left(\boldsymbol{R}, \boldsymbol{R}^{\prime}\right)$ of eqs. (32), (36), and (38), (49), (51) into effective local potentials via the Wigner transform, WKB technique $\left.{ }^{32,33}\right)$, the $G\left(\boldsymbol{R}, \boldsymbol{R}^{\prime}\right)$ must be replaced by their Wigner transforms

$$
G^{\mathrm{W}}\left(\boldsymbol{R}^{2} ; P^{2} ;(\boldsymbol{R} \cdot \boldsymbol{P})^{2}\right) \equiv \int \mathrm{d} s \exp \left[\frac{i}{\hbar}(s \cdot \boldsymbol{P})\right]\left\{\begin{array}{c}
G\left(\boldsymbol{R}-\frac{1}{2} s, \boldsymbol{R}+\frac{1}{2} s\right) \\
G\left(\boldsymbol{R}-\frac{1}{2} s,-\boldsymbol{R}-\frac{1}{2} s\right)
\end{array}\right\},
$$


where the upper/lower factors apply according to whether the coefficient of the $\left(\boldsymbol{R} \cdot \boldsymbol{R}^{\prime}\right)$ term in the exponential factor of the gaussian kernels is positive/negative. [For details see ref. $\left.{ }^{33}\right)$.] Note, however, that the lower factor is required only for certain of the $I_{P, i}^{o}\left(\boldsymbol{R}, \boldsymbol{R}^{\prime}\right)$ in $G_{\alpha}\left(\boldsymbol{R}, \boldsymbol{R}^{\prime}\right)$ with $\alpha \neq 0$.

Finally, the effective local potentials follow from $G^{\mathrm{W}}\left(R^{2} ; P^{2} ;(\boldsymbol{R} \cdot \boldsymbol{P})^{2}\right)$ via the transcendental equation

$$
\begin{aligned}
U^{\mathrm{eff}}(R)= & G^{\mathrm{w}}\left(R^{2} ; M_{\mathrm{N}}\left[E_{\mathrm{c} . \mathrm{m} .}-U^{\mathrm{eff}}(R)\right] ; M_{\mathrm{N}} R^{2}\left[E_{\mathrm{c.m} .}-U^{\mathrm{eff}}(R)\right.\right. \\
& \left.\left.-\frac{\hbar^{2}}{M_{\mathrm{N}} R^{2}}\left(L+\frac{1}{2}\right)^{2}\right]\right)
\end{aligned}
$$

where $L=0$.

\section{Choice of parameters, meson-nucleon coupling constants, and electromagnetic properties}

Since the $(3 q)(q \bar{q})$ components of the nucleon internal functions make significant contributions to the $\mathrm{N}$ - and $\Delta$-masses and the nucleon magnetic moments and rms radii, it is necessary to redetermine the numerical values of the parameters $\alpha_{s}, m$, $b$, and $a_{\mathrm{c}}$. In addition, the strengths of the q⿳亠口冖 pair creation excitations can also be related to the nucleon-meson coupling constants. The vector-meson coupling constants, in particular, will be used to impose new constraints on the choice of parameters. Since a simple $0 \mathrm{~s}(\mathrm{q} \overline{\mathrm{q}})$ cluster with the quantum numbers of the pion, $S T(\lambda \lambda)=01(00)$, cannot be expected to be a realistic model for a physical pion, we do not attempt to fit the pion-nucleon coupling constant. Since our simple model can also not be expected to give a good description of the "pion cloud" in the extreme surface region of a nucleon, the predicted proton rms charge radius will be permitted to be somewhat smaller than the experimental value. Before proceeding to the discussion of numerical values it is necessary to relate the meson-nucleon coupling constants to the parameters of our model and to discuss the modifications imposed on nucleon electromagnetic form factors by the $(3 q)(q \bar{q})$ components of the nucleon wave functions.

The meson-nucleon coupling constants have been discussed by Yu and Zhang ${ }^{35}$ ). Since their treatment differs somewhat from ours, and since they do not discuss the interesting tensor/vector coupling constant ratios for the vector mesons, some detail will be given. If the meson-nucleon interactions are rewritten in the shorthand form

$$
\mathscr{H}_{\beta N N}=\int \mathrm{d} x:\left(\bar{\psi}_{\mathrm{N}} \Gamma_{\beta} \psi_{\mathrm{N}}\right) \cdot \phi_{\beta}:
$$

with $\beta=\eta, \pi, \omega, \rho$, and a scalar product in both 4-space and isospin space for 


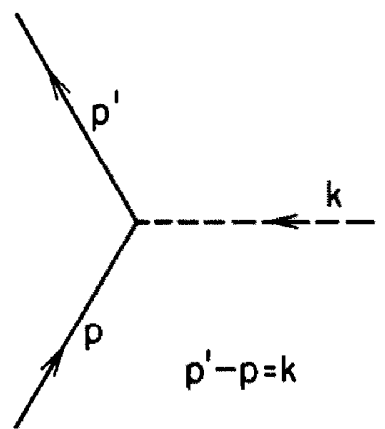

Fig. 3. Nucleon-meson vertex.

meson fields $\phi_{\beta}$ with the appropriate 4-space and isospin scalar/vector characteristics, the relativistic vertex functions are then (see fig. 3 )

$$
\begin{aligned}
\Gamma_{\left(\begin{array}{c}
\eta \\
\pi
\end{array}\right)} & =\sqrt{4 \pi} g_{\left(\begin{array}{c}
\eta \\
\pi
\end{array}\right)} i \gamma^{5}\left(\begin{array}{l}
1 \\
\tau
\end{array}\right), \\
\Gamma_{\left(\begin{array}{c}
\alpha \\
\rho
\end{array}\right)} & =\sqrt{4 \pi}\left[g_{\left(\begin{array}{c}
\omega \\
\rho
\end{array}\right)} \gamma^{\mu}+\frac{1}{2 M_{\mathrm{N}}} f_{\left(\begin{array}{c}
\omega \\
\rho
\end{array}\right)} i \sigma^{\mu \nu} k_{\nu}\right]\left(\begin{array}{l}
1 \\
\tau
\end{array}\right) \\
& =\sqrt{4 \pi}\left[\left(g_{\left(\begin{array}{c}
\omega \\
\rho
\end{array}\right)}+f_{\left(\begin{array}{c}
\omega \\
\rho
\end{array}\right)}\right) \gamma^{\mu}-\frac{1}{2 M_{\mathrm{N}}} f_{\left(\begin{array}{c}
\omega \\
\rho
\end{array}\right)}\left(p+p^{\prime}\right)^{\mu}\right]\left(\begin{array}{l}
1 \\
\tau
\end{array}\right),
\end{aligned}
$$

where the second form for the vector mesons has made use of the Gordon decomposition, assuming the nucleons are on the mass shell. The transition matrix elements $V_{\mathrm{fi}}$, from a nucleon initial state $p$ to a final state $p^{\prime}$ with the emission of a meson of momentum $\boldsymbol{k}$, vector polarization $e^{(\lambda)}$, and isospin orientation $e^{(\nu)}$, can then be expressed in terms of nonrelativistic nucleon vertex functions $H_{\beta}$ by

$$
V_{\mathrm{fi}}=\left\langle m_{s \mathrm{f}} m_{t}\left|H_{\beta}\right| m_{s \mathrm{i}} m_{t \mathrm{i}}\right\rangle \delta_{\mathbf{p}^{\prime}, \boldsymbol{\rho} \cdot \boldsymbol{k}} .
$$

In the nonrelativistic limit, $k / m_{\beta} \ll 1$, and static approximation, $p=0$, the $H_{\beta}$ can be expressed in terms of $\boldsymbol{k}$ and nucleon $\boldsymbol{\sigma}$ and $\boldsymbol{\tau}$ Pauli operators by

$$
\begin{aligned}
H_{\left(\begin{array}{c}
\eta \\
\pi
\end{array}\right)}= & -i g_{\left(\begin{array}{c}
\eta \\
m
\end{array}\right)}\left[\frac{4 \pi}{2 m_{\eta, \pi}}\right]^{1 / 2} \frac{1}{2 M_{\mathrm{N}}}(\boldsymbol{\sigma} \cdot \boldsymbol{k})\left(\begin{array}{c}
1 \\
\boldsymbol{\tau} \cdot \boldsymbol{e}^{(\nu) *}
\end{array}\right), \\
H_{\left(\begin{array}{c}
\omega \\
\rho
\end{array}\right)}= & {\left[\frac{4 \pi}{2 m_{\omega, \rho}}\right]^{1 / 2}\left\{g_{\left(\begin{array}{c}
\omega \\
\rho
\end{array}\right)}\left(\frac{1}{m_{\omega, \rho}}+\frac{1}{2 M_{\mathrm{N}}}\right)\left(\boldsymbol{k} \cdot \boldsymbol{e}^{(\lambda) *}\right)\right.} \\
& +\left(g_{\left(\begin{array}{c}
\omega \\
\rho
\end{array}\right)}+f_{\left(\begin{array}{c}
\omega \\
\rho
\end{array}\right)}\right) \frac{i}{2 M_{\mathrm{N}}}\left([\boldsymbol{\sigma} \times \boldsymbol{k}] \cdot \boldsymbol{e}^{(\lambda) *)}\right\}\left(\begin{array}{c}
1 \\
\boldsymbol{\tau} \cdot \boldsymbol{e}^{(\nu) *}
\end{array}\right) .
\end{aligned}
$$


In our model the above $V_{f_{1}}$ can be related to the matrix elements of the pair-creation interaction $H_{\mathrm{q} \rightarrow \mathrm{qq} \bar{q}}$ between a nucleon initial state $\phi_{0 \mathrm{~s}}(3 \mathrm{q})_{S M_{S} T M_{T}}$, (with $S T=\frac{1}{2} \frac{1}{2}$ and $M_{S} M_{T}=m_{s i} m_{t \mathrm{i}}$ above) and nucleon-meson final state

$$
\mathrm{e}^{-i \boldsymbol{k} \cdot X_{3}} \phi_{0 \mathrm{~s}}(3 \mathrm{q})_{S_{1} M_{S 1}, T_{1} M_{T_{1}}} \times(-i)^{S_{2}+1} \mathrm{e}^{i \boldsymbol{k} \cdot X_{2}} \phi_{0 \mathrm{~s}}(\mathrm{q} \overline{\mathrm{q}})_{S_{2} M_{S 2}, T_{2} M_{r_{2}}}
$$

with $M_{S 2}=\lambda, M_{T 2}=\nu ;(\lambda, \nu=0, \pm 1)$. Note also that the $\phi(3 q), \phi(q \bar{q})$ have the color symmetry $(\lambda \lambda)=(00)$ of real particles, although the color quantum number $(00)$ will be omitted for brevity. (A phase factor has been added to $\phi(q \bar{q})$ to bring the phases of sect. 3 into agreement with standard nucleon-meson phases.) With $\boldsymbol{r}=\boldsymbol{X}_{2}-\boldsymbol{X}_{3}$, the above $V_{\mathrm{fi}}$ can be expressed through eq. (9) by

$$
\begin{aligned}
V_{\mathrm{fi}}= & -\sqrt{4} i^{S_{2}+1}\left(\sqrt{\frac{1}{4}}\left(1-3 P_{34}\right) \mathrm{e}^{i k \cdot r} \phi_{S_{1} M_{S 1} T_{1} M_{T 1}}(3 \mathrm{q}) \phi_{S_{2} M_{S 2} T_{2} M_{T 2}}(\mathrm{q} \overline{\mathrm{q}}) \mid\right. \\
& \times \sum_{i=1}^{3} U_{\mathrm{q} \rightarrow \mathrm{qq} \bar{q}}\left(x_{i} ; x_{4}, x_{5}\right)\left|\phi_{S M_{S} T M_{T}}(3 \mathrm{q})\right\rangle .
\end{aligned}
$$

Due to the vector character of $U_{\mathrm{q} \rightarrow q q \bar{q}}$ only the p-wave component survives in the partial-wave expansion of

$$
\mathrm{e}^{i k \cdot r}=4 \pi \sum_{l m} i^{l} j_{l}(k r) Y_{l m}(\hat{r}) Y_{l m}(\hat{k})^{*}
$$

With some recoupling, and the introduction of the spherical vector component, $k_{m}=\left[{ }_{3}^{4} \pi\right]^{1 / 2} k Y_{1 m}(\hat{k}), V_{\text {fi }}$ can be expressed by

$$
V_{\mathrm{fi}}=i^{S_{2}+1}\left[\frac{3}{4 \pi}\right]^{1 / 2} \sum_{S_{12}, f}(-1)^{S_{12}-S_{1}-T_{1}+T} \frac{\hat{S}}{\hat{S}_{1} \hat{S}_{2} \hat{T}_{2}} U\left(S_{1} S_{2} S 1 ; S_{12} f\right) \bar{\Sigma} V_{\left[S_{1} T_{1} S_{2} T_{2}\right] S_{12}}^{S T}(k),
$$

where

$$
\begin{aligned}
\bar{\Sigma} \equiv \sum_{m} & k_{m}(-1)^{M_{S 2}+M_{T 2}} \frac{\hat{f} \hat{T} \hat{T}_{2}}{\hat{T}_{1}}\left\langle T M_{T} T_{2}-M_{T 2} \mid T_{1} M_{T 1}\right\rangle \\
& \times\left\langle S M_{S} f-\left(M_{S 2}+m\right) \mid S_{1} M_{S 1}\right\rangle\left(f-\left(M_{S 2}+m\right) 1 m\left|S_{2}-M_{S 2}\right\rangle\right. \\
V_{\left[S_{1} T_{1} S_{2} T_{2}\right] S_{12}}^{S T}(k)= & \frac{4 \pi i}{k}\left\langle\left(1-3 P_{34}\right) j_{1}(k r)\left[Y_{1}(\hat{\boldsymbol{r}}) \times\left[\phi_{S_{1} T_{1}}(3 q) \times \phi_{S_{2} T_{2}}(q \bar{q})\right]_{S_{12} T}\right]_{S M_{S} T M_{T}}\right| \\
& \times \sum_{i=1}^{3} U_{q \rightarrow q q \bar{q}}\left(x_{i} ; x_{4}, x_{5}\right)\left|\phi_{S M_{S} T M_{T}}(3 q)\right\rangle
\end{aligned}
$$

Except for the fact that the radial part of the $0 \mathrm{p}$ oscillator function has been replaced by $N_{\alpha \alpha}^{1 / 2}(4 \pi / k) j_{1}(k r)$, this is of the form of $H_{\alpha 0}$ of sect. 3 (cf. eqs. (12) and (23)-(26), but note that $(\lambda \lambda)=(00)$ is now implied). For the case where the baryon in both initial and final state is a nucleon, $S T=\frac{1}{2} \frac{1}{2}=S_{1} T_{1}$, the quantity $\bar{\Sigma}$, defined in eq. (64), can be related to matrix elements of nucleon operators $\Gamma_{\mathrm{N}}^{f}$ with spherical tensor character $f(=0$ or 1$)$, where

$$
\bar{\Sigma}=\left\langle\frac{1}{2} M_{S 12} \frac{1}{2} M_{T 1}\left|\Gamma_{\mathrm{N}}^{f}\left(S_{2}, M_{S 2}, T_{2}, M_{T 2}\right)\right| \frac{1}{2} M_{S}^{\frac{1}{2}} M_{T}\right\rangle,
$$


with

$$
\begin{aligned}
\Gamma_{\mathrm{N}}^{f}\left(S_{2}, M_{S 2}, T_{2}, M_{T 2}\right)= & \left\{\delta_{S_{2} 0} \delta_{f 1}\left(-\sqrt{\frac{1}{3}}(\boldsymbol{\sigma} \cdot \boldsymbol{k})\right)+\delta_{S_{2} 1}\left[\delta_{f 0}\left(\boldsymbol{k} \cdot \boldsymbol{e}^{\left(M_{\mathrm{S}_{2}}\right) *}\right)\right.\right. \\
& \left.\left.+\sqrt{\frac{1}{2}} \delta_{f 1} i\left([\boldsymbol{\sigma} \times \boldsymbol{k}] \cdot \boldsymbol{e}^{\left(M_{S_{2}}\right) *}\right)\right]\right\} \cdot\left\{\delta_{T_{2} 0}+\delta_{T_{2} 1}\left(\boldsymbol{\tau} \cdot \boldsymbol{e}^{\left(M_{T 2}\right) *}\right)\right\},
\end{aligned}
$$

and where $\sigma$ and $\tau$ are nucleon operators, as in eq. (59). We thus obtain the following explicit expressions for the $H_{\beta}$ of eq. (58):

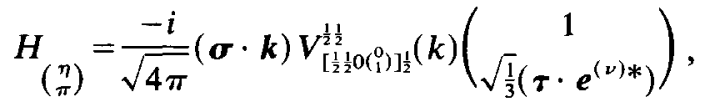

$$
\begin{aligned}
& H_{\left(\begin{array}{l}
\omega \\
\rho
\end{array}\right)}=\frac{1}{\sqrt{4 \pi}}=\frac{1}{\sqrt{3}}\left\{\left(\boldsymbol{k} \cdot \boldsymbol{e}^{(\lambda) *}\right)\left[\boldsymbol{V}_{\left[\frac{1}{2} \frac{1}{2}\left(\begin{array}{l}
0 \\
1
\end{array}\right)\right] \frac{1}{2}}^{\frac{1}{2} \frac{1}{2}}(k)+\sqrt{2} V_{\left.\left[\frac{1}{2} \frac{1}{2} 1\left(\begin{array}{l}
0 \\
1
\end{array}\right)\right]\right]_{2}^{\frac{3}{2}}}^{\frac{11}{1}}(k)\right]\right. \\
& \left.+i\left([\boldsymbol{\sigma} \times \boldsymbol{k}] \cdot \boldsymbol{e}^{(\lambda) *}\right)\left[-V_{\left[\frac{1}{2} \frac{1}{2} 1(\mathbf{1})\right] \frac{1}{2}}^{\frac{11}{2}}(k)+\sqrt{\frac{1}{2}} V_{\left[\frac{1}{2} \frac{1}{2} 1\left(\begin{array}{l}
1 \\
\mathbf{1}
\end{array}\right)\right]_{\frac{1}{2}}^{\frac{1}{2}}}^{(k)}\right]\right\}\left(\begin{array}{c}
1 \\
\sqrt{\frac{1}{3}}\left(\boldsymbol{\tau} \cdot \boldsymbol{e}^{(\nu) *)}\right.
\end{array}\right) .
\end{aligned}
$$

Comparing, eqs. (59) and (67), after restoring the constants $\hbar, c$ (which have tacitly been set to 1 in this discussion up to now), and defining (with $m_{\beta}=$ meson mass, $m=$ quark mass)

$$
A_{\beta} \equiv \frac{1}{\pi}\left[\frac{m_{\beta}}{2 m}\right]^{1 / 2}\left(\frac{M_{\mathrm{N}}}{m}\right)\left(\frac{m c}{\hbar}\right)^{3 / 2} \frac{1}{\hbar c},
$$

the meson-nucleon coupling constants (in units of $\sqrt{\hbar c}$ ) are given by

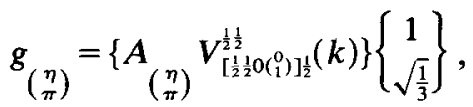

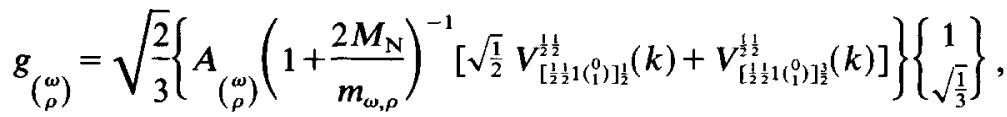

$$
\begin{aligned}
& g_{\left(\begin{array}{c}
\omega \\
\rho
\end{array}\right)}+f_{\left(\begin{array}{c}
\omega \\
\rho
\end{array}\right)}=\sqrt{\frac{1}{6}} A_{\left(\begin{array}{c}
\omega \\
\rho
\end{array}\right)}\left\{-\sqrt{2} V_{\left[\frac{1}{2} \frac{1}{2} 1\left(\begin{array}{l}
0 \\
1
\end{array}\right)\right] \frac{1}{2}}^{\frac{1}{2}}(k)+V_{\left[\frac{1}{2} \frac{1}{2} 1\left(\begin{array}{l}
0 \\
1
\end{array}\right)\right] \frac{1}{2}}^{\frac{11}{2}}(k)\right\}\left\{\begin{array}{c}
1 \\
\sqrt{\frac{1}{3}}
\end{array}\right\},
\end{aligned}
$$

where in the low-energy limit $(k=0)$

$$
\begin{aligned}
A_{\beta} V_{\alpha}^{S T}(k=0)= & {\left[\frac{m_{\beta}}{m}\right]^{1 / 2}\left(\frac{M_{\mathrm{N}}}{m}\right) \frac{\alpha_{\mathrm{s}}}{\sqrt{x}}\left(\frac{\pi}{2}\right)^{3 / 4} \frac{12 \sqrt{2}}{5 \pi} \frac{\hat{S}_{12}}{\hat{S}} } \\
& \times\left[\frac{2}{5}\left(\frac{62}{13} \sqrt{\frac{1}{13}}+\frac{17}{3} \sqrt{\frac{1}{3}}\right) X_{(34), 3}^{\mathrm{M}, \alpha}-\frac{32}{13} \sqrt{\frac{1}{13}} X_{(34), 2}^{\mathrm{D}, \alpha}+\frac{1}{3} \sqrt{\frac{1}{3}} X_{(34), 3}^{\mathrm{D}, \alpha}\right]
\end{aligned}
$$

The spin, isospin, color factors $X_{p, i}^{Q, \alpha}$, with $\alpha=\left[S_{1} T_{1}(00) S_{2} T_{2}(00)\right] S_{12}$, are given as part of table $1 \mathrm{a}$. It is also interesting to note that the ratios $\left(V_{\alpha}(k=0) / V_{\alpha^{\prime}}(k=0)\right)$ are almost identical to the ratios $\left(H_{\alpha 0} / H_{\alpha^{\prime} 0}\right)$, the largest difference being $\sim 3 \%$. 
Finally, the static electromagnetic properties of the nucleon will be extracted from the low- $q$ limits of the electromagnetic form factors which follow from the nucleon electromagnetic current $J$ via

$$
J_{\mathrm{fi}}=\left\langle P_{\boldsymbol{k}^{\prime}} \psi_{\mathrm{N}}\left|j_{\mathrm{e} . \mathrm{m}}\right| P_{k} \psi_{\mathrm{N}}\right\rangle,
$$

where $j_{\text {e.m. }}$ is the quark current operator and where $\left|P_{k} \psi_{N}\right\rangle$ is given by eq. (10). By choosing the Breit frame, with $k=-\frac{1}{2} q, k^{\prime}=+\frac{1}{2} q$, but using the $k$-independent approximation for the $c_{0}, c_{\alpha}$, the form factors follow from

$$
\begin{aligned}
& J_{\mathrm{fi}}(-q)=\left\langle\mathrm{e}^{(i / 2) \boldsymbol{q} \cdot x_{3}} \phi_{0}\left|j_{\text {e.m. }}(-q)\right| \mathrm{e}^{-(i / 2) q \cdot x_{3}} \phi_{0}\right\rangle \\
& +\sum_{\alpha \neq 0} c_{0} c_{\alpha}\left[\left(\mathrm{e}^{(i / 2) \boldsymbol{q} \cdot x_{5}} \phi_{\alpha}\left|j_{\mathrm{e} . \mathrm{m} .}(-q)\right| \mathrm{e}^{-(i / 2) \boldsymbol{q} \cdot \boldsymbol{x}_{3}} \phi_{0}\right\rangle\right. \\
& \left.+\left\langle\mathrm{e}^{(i / 2) \boldsymbol{q} \cdot \boldsymbol{x}_{3}} \phi_{0}\left|j_{\mathrm{e} . \mathrm{m} .}(-\boldsymbol{q})\right| \mathrm{e}^{-(i / 2) \boldsymbol{q} \cdot \boldsymbol{x}_{5}} \phi_{\alpha}\right\rangle\right] \\
& +\sum_{\alpha, \alpha^{\prime} \neq 0} c_{\alpha} c_{\alpha^{\prime}}\left[\left\langle\mathrm{e}^{(i / 2) \boldsymbol{q} \cdot \boldsymbol{x}_{s}} \phi_{\alpha}\left|j_{\text {e.m. }}(-\boldsymbol{q})\right| \mathrm{e}^{-(i / 2) \boldsymbol{q} \cdot \boldsymbol{x}_{s}} \phi_{\alpha^{\prime}}\right\rangle\right. \\
& \left.-\left\langle\phi_{\alpha} \mid \phi_{\alpha}\right\rangle\left\langle\mathrm{e}^{(i / 2) \boldsymbol{q} \cdot \boldsymbol{X}_{3}} \phi_{0}\left|j_{\text {e.m. }}(-\boldsymbol{q})\right| \mathrm{e}^{-(i / 2) \boldsymbol{q} \cdot \boldsymbol{X}_{3}} \phi_{0}\right\rangle\right],
\end{aligned}
$$

where $c_{0}^{2}$ has been eliminated from the dominant term via the normalization condition, eq. (27). In the dominant $3 q$ term and the important cross-term the full relativistic quark current operator is to be used,

$$
j_{\text {e.m. }}^{\mu}(-\boldsymbol{q})=\int \mathrm{d} x \mathrm{e}^{\mathrm{iq} \cdot \boldsymbol{x}}:\left(\bar{Q}(\boldsymbol{x}) \gamma^{\mu}\left(\frac{1}{6}+\frac{1}{2} \tau_{z}\right) Q(\boldsymbol{x})\right):
$$

Note that the quark field operators $\bar{Q}(x), Q(x)$ lead to both $a^{+} a, b^{+} b$, as well as $a^{+} b^{+}, b a$ terms in $j_{\text {e.m. }}(-q)$. Since the second-order $c_{\alpha} c_{\alpha}^{i}$ term is small a nonrelativistic approximation can here be used for $j_{\text {e.m. }}$. Details for the calculation of the electromagnetic form factors will be given elsewhere. It should be pointed out, however, that by far the largest contributions to the $c_{0} c_{\alpha}$ cross-terms arise from direct matrix elements which can exist only for the vector-meson terms with $\alpha=N \omega S_{12}$ and $\alpha=N \rho S_{12}$. Approximate formulae containing the dominant $3 \mathrm{q}$ term and the direct vector-meson matrix elements only are therefore quite simple. Again with $x=\hbar / m c b$, the charge $\left\langle r^{2}\right\rangle$ and magnetic moment expressions containing only these terms are given by

$$
\begin{aligned}
& \left.\begin{array}{l}
\left\langle r^{2}\right\rangle_{\mathrm{p}} / b^{2} \\
\left\langle r^{2}\right\rangle_{\mathrm{n}} / b^{2}
\end{array}\right\} \simeq\left\{\begin{array}{c}
1+\frac{3}{4} x^{2} f^{(1)}(x) \\
0
\end{array}\right\}-x_{3}^{8}\left(\frac{5}{3}\right)^{1 / 4} c_{0} g^{(2)}(x)\left\{\begin{array}{c}
A_{\omega}+\sqrt{3} A_{\rho} \\
A_{\omega}-\sqrt{3} A_{\rho}
\end{array}\right\},
\end{aligned}
$$

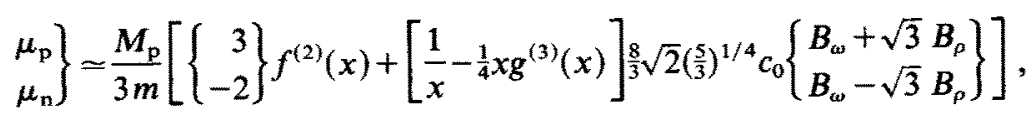


where the $A_{\omega}, \ldots, B_{\rho}$ are given in terms of the $c_{\alpha}$ by

$$
\begin{gathered}
A_{\left(\begin{array}{c}
\omega \\
\rho
\end{array}\right)}=\sqrt{\frac{1}{3}} c_{\mathrm{N}\left(\begin{array}{c}
\omega \\
\rho
\end{array}\right) \frac{1}{2}}+\sqrt{\frac{2}{3}} c_{\mathrm{N}\left({ }_{\rho}^{\omega}\right)^{\frac{3}{2}}}, \\
B_{\left(\begin{array}{c}
\omega \\
\rho
\end{array}\right)}=\sqrt{\frac{2}{3}} c_{\mathrm{N}\left(\begin{array}{c}
\omega \\
\rho
\end{array}\right) \frac{1}{2}}-\sqrt{\frac{1}{3}} c_{\mathrm{N}\left(\begin{array}{c}
\omega \\
\rho
\end{array}\right)^{\frac{3}{2}}},
\end{gathered}
$$

and where $f^{(i)}(x), g^{(i)}(x)$ are relativistic reduction factors normalized such that $f^{(i)}(0)=g^{(i)}(0)=1$,

$$
\left.\begin{array}{l}
f^{(i)}(x) \\
g^{(2)}(x) \\
g^{(3)}(x)
\end{array}\right\}=\frac{2}{\sqrt{\pi}} \int_{0}^{\infty} \mathrm{d} t \sqrt{t} \mathrm{e}^{-t}\left\{\begin{array}{c}
F^{(i)}\left(\left[1+\frac{2}{3} x^{2} t\right]^{1 / 2}\right) \\
F^{(2)}\left(\left[1+x^{2} t\right]^{1 / 2}\right) \\
\frac{2}{3} t F^{(3)}\left(\left[1+x^{2} t\right]^{1 / 2}\right)
\end{array}\right\},
$$

with

$$
\begin{aligned}
& F^{(1)}(a)=\frac{2}{a(1+a)}\left[1-\frac{1}{6}\left(1-\frac{1}{a}\right)\left(2+\frac{2}{a}+\frac{1}{a^{2}}\right)\right], \\
& F^{(2)}(a)=\frac{1}{3 a}\left(2+\frac{1}{a}\right), \\
& F^{(3)}(a)=\frac{2}{a(a+1)} .
\end{aligned}
$$

The full results including all terms of eq. (72) are given in table 4 below.

With eqs. (69) and (72) and the single-baryon energies evaluated in sect. 3, a careful reevaluation of the model parameters $\alpha_{\mathrm{s}}, m, b, a_{\mathrm{c}}$ can now be made. The philosophy for the choice of the numerical values has been outlined in the introduction. The following experimental quantities are to be fitted:

(i) the $M_{\Delta}-M_{\mathrm{N}}$ mass difference $(293 \mathrm{MeV})$;

(ii) the vector-meson coupling constants; in particular, the combination $\left(m_{\rho} / 2 M_{\mathrm{N}}\right)\left(g_{\rho}+f_{\rho}\right)$, with experimental value in the range 1.97-2.49, which ensures a fit for the corresponding $\omega$-coupling constants (see table 3 below);

(iii) the nucleon magnetic moments, specifically the isovector component $\frac{1}{2}\left(\mu_{\mathrm{p}}-\right.$ $\mu_{n}$ ), experimental value 2.353 , to avoid sensitivity to small variations in the difference of absolute values.

Finally, the nucleon mass itself is fitted. This is achieved by the choice of $a_{\mathrm{c}}$, the confining potential constant. Since the final NN scattering results, as well as the quantities (i) to (iii) are very insensitive to the value of $a_{c}$, this last choice is made largely for convenience so that the real nucleon mass, $939 \mathrm{MeV}$, can be used in subsequent calculations. In addition, the rms radius of the proton is examined, although its predicted value is expected to be somewhat less than the experimental one. 


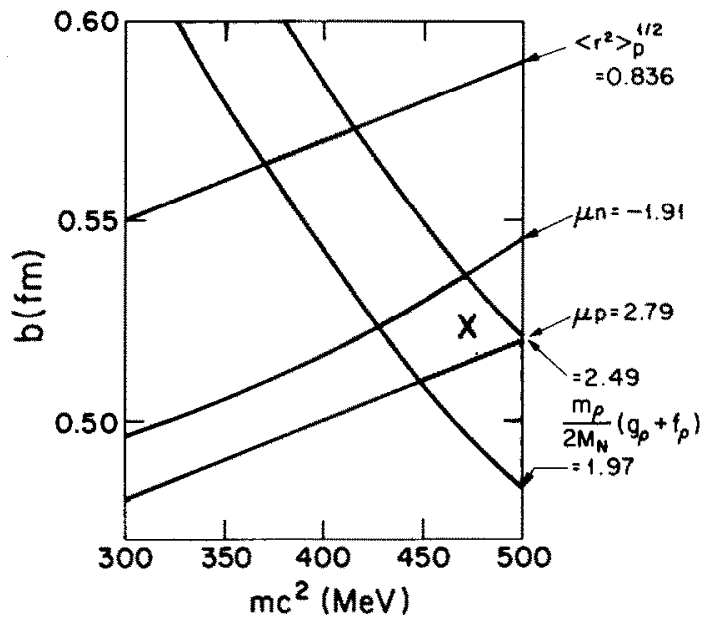

Fig. 4. The $b$ versus $m$ plot used to fit the model parameters; with $\alpha_{s}, a_{c}$ chosen to fit $M_{\Delta}-M_{\mathrm{N}}$ and $M_{\mathrm{N}}$. The cross indicates the final parameter choice (see first entry of table 2).

TABLE 2

The model parameters

\begin{tabular}{llllll}
\hline & $b[\mathrm{fm}]$ & $m c^{2}[\mathrm{MeV}]$ & $\alpha_{\mathrm{s}}$ & $a_{\mathrm{c}}\left[\mathrm{MeV} \cdot \mathrm{fm}^{-2}\right]$ & $x$ \\
\hline present & 0.5235 & 471.2 & 2.973 & 335.7 & 0.8 \\
q model $^{\mathrm{a}}$ ) & 0.6 & 313 & 1.517 & 23.67 & 1.051 \\
\hline
\end{tabular}

a) Ref. ${ }^{17}$ ).

The fitting procedure is carried out as follows. The constants $\alpha_{\mathrm{s}}$ and $a_{\mathrm{c}}$ are chosen first to fit $M_{\Delta}-M_{\mathrm{N}}$ and $M_{\mathrm{N}}$. The remaining quantities $\mu_{\mathrm{p}}, \mu_{\mathrm{n}},\left\langle r^{2}\right\rangle_{\mathrm{p}}^{1 / 2}$, and the $\rho \mathrm{N}$ coupling constants are then examined on a $b$ versus $m$ plot. Fig. 4 shows such a plot to illustrate the sensitivity of the fitted quantities to these parameters. The final values of the parameters are shown in table 2 (first entry). These are contrasted

TABLE 3

Meson-nucleon coupling constants

\begin{tabular}{|c|c|c|c|c|c|c|}
\hline \multirow{2}{*}{$\beta$} & \multicolumn{2}{|c|}{$g_{\beta}^{2}$} & \multicolumn{2}{|c|}{$f_{\beta} / g_{\beta}$} & \multicolumn{2}{|c|}{$\left(m_{\beta} / 2 M_{N}\right) \cdot\left(g_{\beta}+f_{\beta}\right)$} \\
\hline & theor. & $\left.\exp ^{a}\right)$ & theor. & $\left.\exp .^{a}\right)$ & theor. & $\exp { }^{a}$ ) \\
\hline$\left.\eta^{b}\right)$ & 6.89 & $3.7-7.6$ & - & - & 0.77 & $0.6-0.8$ \\
\hline$\pi$ & 4.70 & $13.7-14.4$ & - & - & 0.16 & $0.27-0.28$ \\
\hline$\omega$ & 9.65 & $7.9-14.6$ & 0.18 & $0-0.7$ & 1.52 & $1.4-2.4$ \\
\hline$\rho$ & 1.26 & $0.4-0.8$ & 3.98 & $4.3-6.6$ & 2.31 & $2.0-2.5$ \\
\hline
\end{tabular}

a) Ref. ${ }^{41}$ ).

b) $m_{\eta} c^{2}=549 \mathrm{MeV}$ is used. 
TABLE 4

Nucleon electromagnetic properties

\begin{tabular}{|c|c|c|c|c|c|c|c|c|c|}
\hline & & $3 q$ & & Cross-term & & $\begin{array}{l}\text { Second-order } \\
\text { term }\end{array}$ & & Total & Exp. $\left.{ }^{41}\right)$ \\
\hline$\left\langle r^{2}\right\rangle_{\mathrm{p}} / b^{2}$ & $=$ & 1.306 & + & 0.497 & + & 0.144 & $=$ & 1.947 & \\
\hline$\left\langle r^{2}\right\rangle_{\mathbf{p}}^{1 / 2}$ & $=$ & 0.598 & $\rightarrow$ & 0.703 & $\rightarrow$ & $\left.0.731^{a}\right)$ & & & $\left.0.836^{a}\right)$ \\
\hline$\left\langle r^{2}\right\rangle_{n} / b^{2}$ & $=$ & 0 & - & 0.012 & - & 0.009 & $=$ & -0.021 & \\
\hline$\left|\left\langle r^{2}\right\rangle_{n}\right|^{1 / 2}$ & $=$ & 0 & $\rightarrow$ & 0.06 & $\rightarrow$ & $0.08^{\mathrm{a}}$ ) & & & $\left.0.34^{\mathrm{a}}\right)$ \\
\hline$\mu_{\mathrm{p}}$ & $=$ & 1.51 & + & 1.32 & + & 0.00 & $=$ & 2.83 & 2.79 \\
\hline$\mu_{n}$ & $=$ & -1.01 & - & 0.86 & - & 0.01 & $=$ & -1.88 & -1.91 \\
\hline$g_{\mathrm{A}} / g_{\mathrm{V}}$ & $=$ & 1.45 & + & 0.36 & + & 0.05 & $=$ & 1.86 & 1.26 \\
\hline
\end{tabular}

a) Units: fm.

with an earlier set determined for a pure $3 q$ model of the nucleon, using a similar fitting philosophy ${ }^{17}$ ).

With the final choice of parameters it is now possible to examine the mesonnucleon coupling constants and the nucleon electromagnetic properties in more detail. The meson-nucleon coupling constants are shown in table 3. Both the $\omega$ and $\rho$-coupling constants fall within the range of values extracted from the experimental nucleon scattering data. It is encouraging to note that the model fits the large differences in the tensor/vector ratios $f / g$ for $\omega$ and $\rho$. Since the $\eta$-meson of our model is made up of nonstrange quarks only, a mass somewhere between that of the physical $\eta$ and $\eta^{\prime}$ should perhaps be used for the comparison. The results for $g_{\eta}$ are acceptable. As expected, our predicted value for $g_{\pi}$ is too weak $\left(g_{\pi}^{2}\right.$ is $-33 \%$ of the experimental value). It should also be noted that higher-order contributions in the pair-creation interaction and other small improvements in the model, examined by $\mathrm{Yu}$ and $\mathrm{Zhang}{ }^{35}$ ), do not make essential changes in these results.

Table 4 shows the predicted values for the static electromagnetic quantities. The contributions of the $3 q$ term, the cross-terms and the second-order terms of eq. (72) are shown separately. It is interesting to note that the proton rms radius, which is increased from 0.523 to $0.598 \mathrm{fm}$ by the relativistic correction term, is further increased to $0.731 \mathrm{fm}$ by the $(3 q)(q \bar{q})$ components of our model. These make important contributions to all the electromagnetic properties. The model fails to give the experimental neutron charge $\left\langle r^{2}\right\rangle$ value. It has been suggested by Oka and Yazaki ${ }^{42}$ ) that a small $2 \hbar \omega$ excitation of space symmetry [21] and color singlet character in the $3 \mathrm{q}$ model of the nucleon is vital for this quantity. Using their estimate for the amplitude of this excitation $\left(c_{[21]}=-0.297\right)$, a simple perturbation estimate gives $\Delta\left(\left\langle r^{2}\right\rangle / b^{2}\right) \sim \pm 2 \sqrt{\frac{1}{6}}(0.297)\left[1-(0.297)^{2}\right]^{1 / 2}= \pm 0.23$ for $\mathrm{p} / \mathrm{n}$. This would increase $\sqrt{\left\langle r^{2}\right\rangle_{p}}$ from 0.73 to $0.77 \mathrm{fm}$ and $\left|\left\langle r^{2}\right\rangle_{\mathrm{n}}\right|^{1 / 2}$ from 0.08 to $0.26 \mathrm{fm}$. In view of the expected failure of our model in the extreme surface region of the nucleon these may be reasonable predictions. Table 4 includes the predicted value for the ratio of axial vector/vector coupling constants. Although the relativistic correction factor 
decreases the $3 q$ contribution, from the value of $\frac{5}{3}$, this decrease is more than compensated by the contributions of the $(3 q)(q \bar{q})$ components.

The crucial numbers for our model are the constants $c_{\alpha}$ which give the magnitudes of the $(3 q)(q \bar{q})$ components of the nucleon wave function. These are shown in table 1 as determined from the present parameter set (first entry of table 2). They lie in the $0-0.25$ range. The very largest amplitudes are those for states with color $\mathrm{SU}(3)$ singlet character and with $S_{12}=\frac{3}{2}$, i.e. with $\alpha=\mathrm{N} \omega, \mathrm{N} \rho, \Delta \pi$ and $\Delta \rho$, and $S_{12}=\frac{3}{2}$; the next largest being those for $\alpha=\mathrm{N} \pi, \mathrm{N} \rho$ and $\Delta \rho$, and $S_{12}=\frac{1}{2}$. The preference for $S_{12}=\frac{3}{2}$ appears to be related to the predominance of the $\mathrm{D}$ (derivative) type term in the pair-creation interaction. Note that the D-term of $\mathbf{H}_{\mathrm{q} \rightarrow \mathrm{qq} \bar{q}}$ acts only in states with a qqā spin of $\frac{3}{2}$ (cf. eq. (5)). The amplitudes of the hidden color components lie in the 0-0.05 range and are much smaller than those for the $(\lambda \lambda)=(00)$ states. Of the hidden color states, the channels with an $\omega^{c}$ appear to have the largest amplitudes. Since the $\omega^{c}$ carries the gluon quantum numbers these amplitudes can get a contribution from a direct term. Despite the much smaller amplitudes of the hidden color channels it appears that these have an important coherent effect on the NN interaction (see sect. 5). The value of $c_{0}=0.857$ shows that the (3q) $(\mathrm{q} \overline{\mathrm{q}})$ components carry about $27 \%$ of the nucleon wave function.

The question arises as to whether the numerical values of the $c_{\alpha}$ are very sensitive to the exact choice of the parameters $\alpha_{\mathrm{s}}, m, b$, and $a_{\mathrm{c}}$ or to the nature of the quark internal wave functions. An evaluation of the $c_{\alpha}$ with the earlier 3q parameter set of table 2 (despite the major difference in the parameter $a_{\mathrm{c}}$ ), gives a set of $c_{\alpha}$ 's almost identical with those for the parameter set of the present investigation. For example, the four largest $c_{\alpha}$ 's with $S_{12}=\frac{3}{2}$, are now: -0.230 for $\mathrm{N} \omega \frac{3}{2},-0.258$ for $\mathrm{N} \rho \frac{3}{2}, 0.202$ for $\Delta \pi \frac{3}{2},-0.252$ for $\Delta \rho_{2} \frac{3}{2}$, cf. table 1a; whereas with $S_{12}-\frac{1}{2}$, the largest $c_{a}$ 's are now: -0.190 for $\mathrm{N} \pi \frac{1}{2}, 0.138$ for $\mathrm{N} \rho \frac{1}{2}$, and -0.107 for $\Delta \rho \frac{1}{2}$. The value of $c_{0}$ is 0.852 (compared with 0.857 for the parameter set of the present investigation), so that the percentage of $(3 q)(q \bar{q})$ components are essentially identical for the two parameter sets.

Finally, to test the importance of relativistic effects for the orders of magnitude of the $c_{\alpha}$, the $\phi_{\alpha}$ of eq. (12) have been expanded in a $\mathrm{q}^{4} \overline{\mathrm{q}}$ quark shell-model basis. The resultant amplitudes have been compared with a relativistic calculation in which zero-rest mass MIT bag 0s and $0 \mathrm{p}$ wave functions were used. This relativistic calculation gives similar but slightly smaller amplitudes. The values of the $c_{\alpha}$ thus appear to be quite insensitive to the details of the model. In particular, they pass the crucial test of being insensitive to large changes in the parameter $a_{\mathrm{c}}$.

\section{The effective NN potentials}

The Wigner transforms of the quark exchange kernels $G\left(R, R^{\prime}\right)$, in the approximation of eq. (32), have been used to evaluate phase-shift-equivalent local potentials 


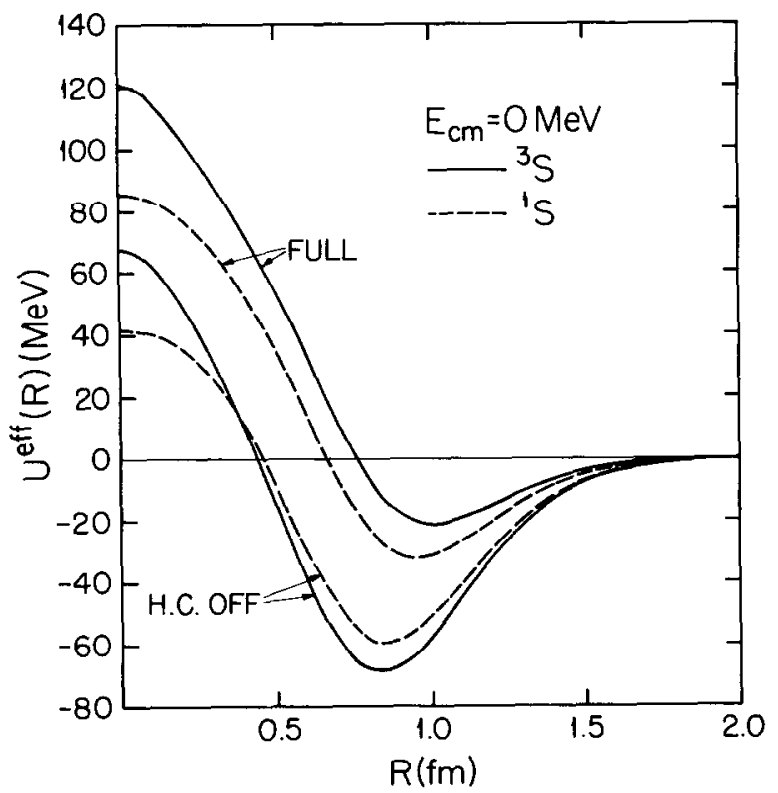

Fig. 5. The S-wave phase-shift-equivalent local potentials for $E_{\text {c.m. }}=0$. The curves show the results for both the full calculation and for a calculation with hidden color states turned off.

through the self-consistency equation, (55). Such effective local potentials for S-wave scattering are shown in fig. 5 for the extreme low-energy limit, $E_{\text {c.m. }}=0$. The admixtures of $(3 q)(q \bar{q})$ components into the nucleon wave functions lead to two prominent effects. The repulsive core heights, which would have been $\sim 700-900 \mathrm{MeV}$ in a pure $3 q-3 q$ model, with our interaction, are greatly reduccd to around $100 \mathrm{MeV}$; and the potentials have gained a weak attractive part in the $0.8-1.5 \mathrm{fm}$ range. The ${ }^{1} \mathrm{~S}$ potential, with a minimum of $\sim-32 \mathrm{MeV}$, is slightly more attractive than the ${ }^{3} \mathrm{~S}$ potential, with a minimum of $\sim-21 \mathrm{MeV}$. Fig. 5 also shows the effective potentials obtained when the 15 hidden color channels are arbitrarily turned off. The potentials become more attractive, with minima of $-59 \mathrm{MeV}$ for ${ }^{1} \mathrm{~S}$ and $-69 \mathrm{MeV}$ for ${ }^{3} \mathrm{~S}$. The hidden color channels play a significant role in making the potentials more repulsive. Fig. 6, which gives the effective local potentials in the $0-350 \mathrm{MeV}$ range (NN scattering $\mathrm{cm}$ energies), shows that the strong energy dependence of the repulsive cores is similar to that of the pure $3 q-3 q$ models. The repulsive core heights are now very similar to those for the phenomenological short-range terms introduced into the Paris potential ${ }^{43}$ ). This is illustrated in fig. 7 which summarizes the energy dependence of the core heights and the potential depths of the attractive part of the NN potentials. The repulsive core heights predicted by the full calculation are now in good agreement with the Paris potential in the $200-300 \mathrm{MeV}$ region where our quark model may be expected to have its greatest validity.

Although our model leads to a medium-range attraction, this attraction is too weak to bind the deuteron or explain the experimental low-energy scattering phase 


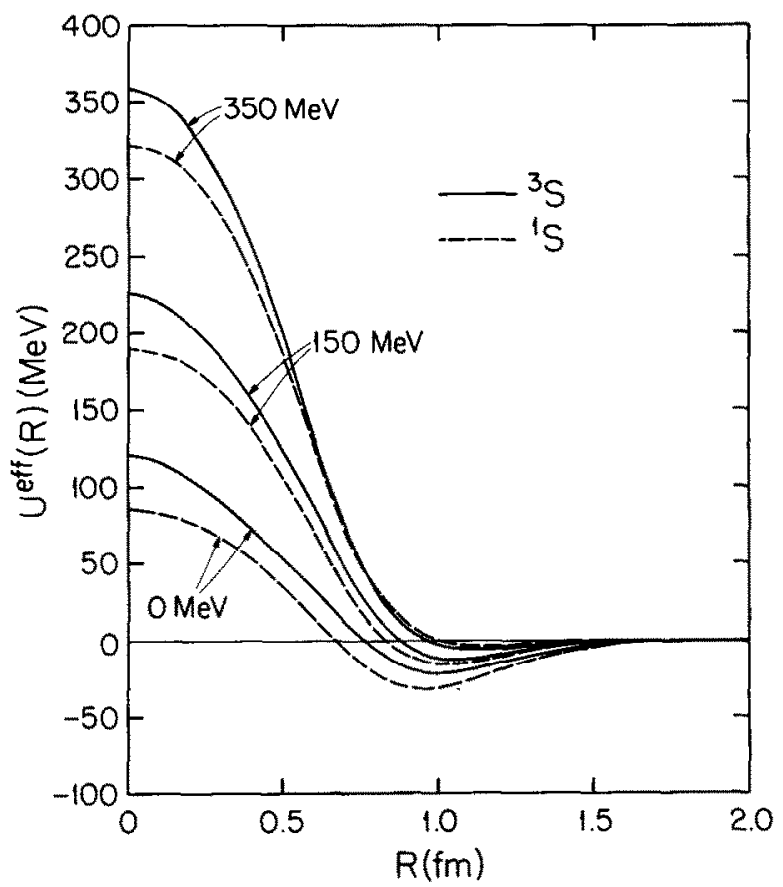

Fig. 6. The energy dependence of the effective local potentials in the $0-350 \mathrm{MeV}$ range (NN scattering $\mathrm{cm}$ energies).

shifts. This is shown in fig. 8 which gives the S-wave phase shifts for our effective potentials. With all $(3 q)(q \bar{q})$ components turned off the S-wave phase shifts are purely repulsive. For the full calculation the extreme low-energy phase shifts show a weak attraction. With the hidden color channels turned off, the low-energy attractive phase shifts are closer to the experimental values with the ${ }^{3} \mathrm{~S}$ more attractive than the ${ }^{1} S$, seemingly in better agreement with experiment. However, the phase shifts with hidden color states turned off are not sufficiently repulsive in the $200-300 \mathrm{MeV}$ range. At these energies the phase shifts for the full calculation match the experimental quantities both in absolute value and in the near-equality of $\delta\left({ }^{3} \mathrm{~S}\right)$ and $\delta\left({ }^{1} \mathrm{~S}\right)$. Since phase shifts in this energy region might be expected to be least sensitive to further improvements in our quark model, we conclude that the repulsive effect of the hidden color states is an important ingredient of our model. Clearly, also, improvements in the model are needed for the low-energy scattering domain or the long-range part of the NN interaction. The phase shifts in fig. 8 have been calculated with our equivalent local potentials. However these have also been checked against single-channel S-wave RGM calculations using the exchange kernels directly, with reasonable agreement between the two types of calculations. (Details of the full RGM calculations will be given elsewhere.)

To gain a better understanding of the effective NN potentials it is interesting to examine the relative importance of the various $(3 q)(q \bar{q})$ components. The Wigner 


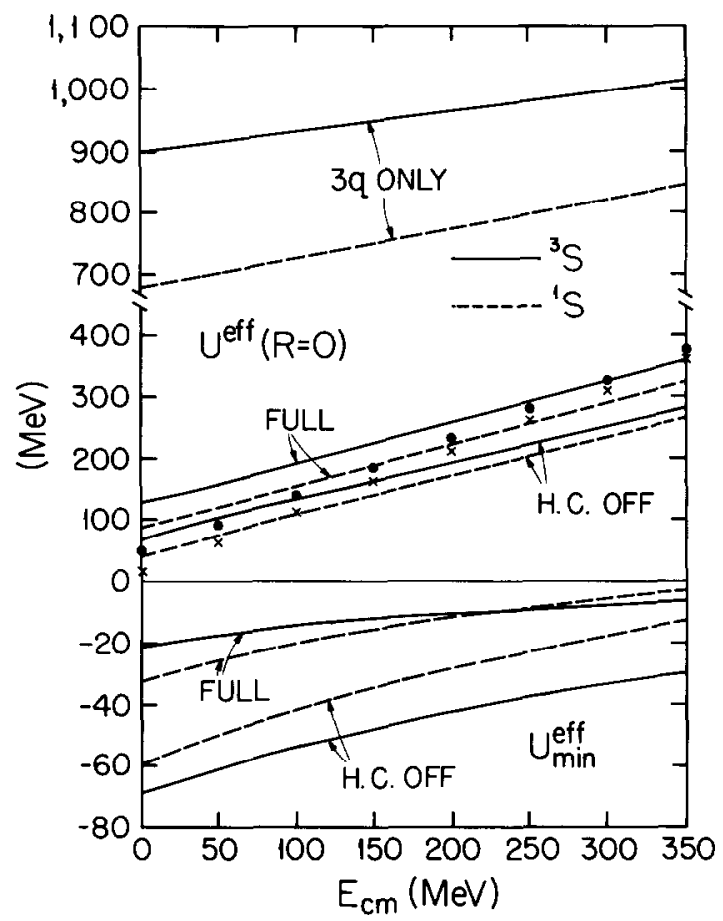

Fig. 7. Energy dependence of the repulsive core heights and potential minima. The repulsive core heights for a simple $3 q-3 q$ model are greatly reduced by the $(3 q)(q \bar{q})$ terms. Results for both the full calculation and a calculation with hidden color states turned off are shown. The dots $\left({ }^{3} \mathrm{~S}\right)$ and crosses $\left({ }^{1} \mathrm{~S}\right)$ give the repulsive core heights of the phenomenological short-range terms of the Paris potential ${ }^{43}$ ).

transforms of the exchange kernels are ideal for this purpose. Since the cross-terms are linear in the $c_{\alpha}$ it is possible to turn on one term at a time and examine the relative strengths and radial dependences of individual $c_{0}^{3} c_{\alpha} G_{\alpha}^{\mathrm{W}}\left(R ; q^{2}\right)$ contributors. For this purpose it is useful to use the dimensionless momentum variable $q^{2}=$ $\left(2 \dot{M}_{N} b^{2} / 3 \hbar^{2}\right)\left(E_{\text {c.m. }}-U^{\text {eff }}(R)\right)$. For the parameters of this investigation, $\left|q^{2}\right|=1$ corresponds to an energy of $\sim 225 \mathrm{MeV}$. For $E_{\text {c.m. }}$ in the $0-300 \mathrm{MeV}$ range and the $U^{\text {eff }}$ of figs. 5 and 6 , therefore, it can be seen that the $q^{2}$ values range from $q^{2} \simeq+1$ for very large $R$ to $q^{2} \simeq 0$ as $R \rightarrow 0$, and the Wigner transforms $G_{\alpha}^{\mathrm{w}}$ for $q^{2}=0$ should give a good indication of the nature of a particular "baryon-meson" term in the $R=0-1 \mathrm{fm}$ range. Fig. 9 shows the $c_{0}^{3} c_{\alpha} G_{\alpha}^{\mathrm{W}}\left(R, q^{2}=0\right)$ for the three states $\alpha$ with $S_{12}=\frac{1}{2}$ and $(\lambda \lambda)=(00)$ and the largest values of $c_{\alpha}$, i.e. the states $\alpha=\mathrm{N} \pi \frac{1}{2}, \mathrm{~N} \rho \frac{1}{2}$, and $\Delta \rho \frac{1}{2}$. Although all give attractive contributions to the potentials in the $R \approx 1 \mathrm{fm}$ region, the much larger contributions as $R \rightarrow 0$ include both attractive and strong repulsive pieces. Figs. 10 and 11 show similar results for the four states $\alpha$ with $S_{12}=\frac{3}{2}$ which have the dominant amplitudes $c_{\alpha}$. Although the $\mathrm{N} \omega \frac{3}{2}$ component (fig. 10) gives a weak repulsive contribution in the $R=1-1.5 \mathrm{fm}$ range, its strong attraction as $R \rightarrow 0\left(-1187 \mathrm{MeV} R=0\right.$ value for ${ }^{1} \mathrm{~S},-1319 \mathrm{MeV}$ for $\left.{ }^{3} \mathrm{~S}\right)$ helps to overpower 


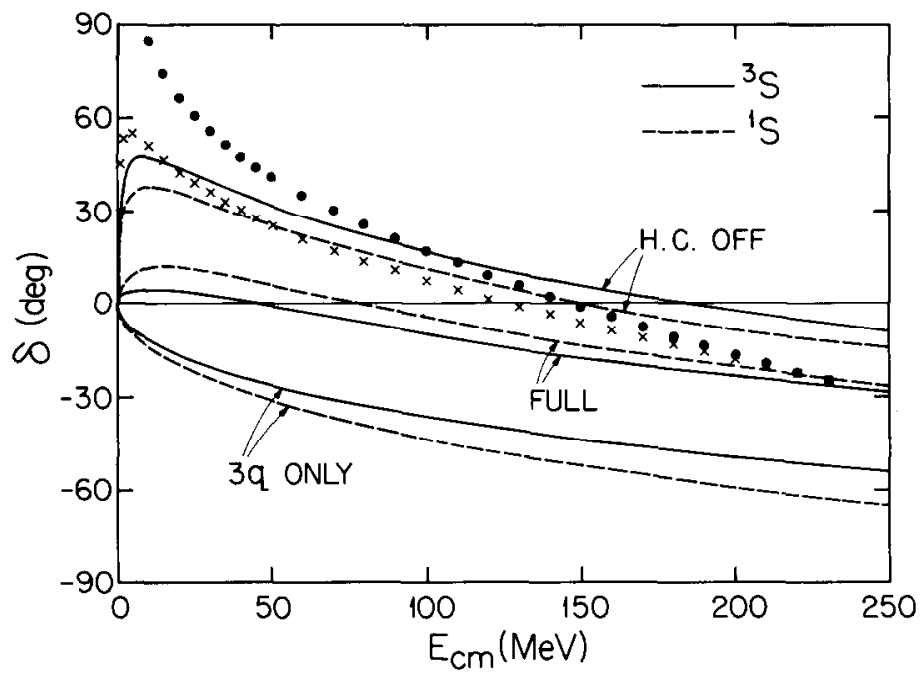

Fig. 8. S-wave phase shifts for the effective potentials. Results are shown for the full calculation, and a calculation with hidden color states turned off, also for a calculation with $3 q$ components only. The dots $\left({ }^{3} \mathrm{~S}\right)$ and crosses $\left({ }^{1} \mathrm{~S}\right)$ give the experimental phase shifts $\left.{ }^{44}\right)$.

some of the strong repulsive contributions such as those of the $\Delta \pi_{2}^{\frac{3}{2}}$ and $\Delta \rho \frac{3}{2}$ channels of fig. 11. It should perhaps be strongly emphasized at this point that the baryonmeson names based on our RGM two-cluster wave functions $\phi_{\alpha}$ may have physical significance in terms of real mesons only in the large- $R$ limit, but lose this significance as $R \rightarrow 0$ since antisymmetrization and recoupling mix up all 24 components $\phi_{\alpha}$ in this domain. Nevertheless, it is encouraging that the dominant $\mathrm{N} \omega$ components lead to repulsion in the large- $R$ limit. Although the 15 hidden color components (not shown in figs. 9-11) also have both attractive and repulsive contributors their overall effect is more coherent and they lead to the net repulsive effect illustrated in fig. 5 . The net attractive effect of all 24 cross-terms is shown in fig. 12 which gives the full cross-term contributions to the Wigner transforms of the exchange kernels for the $q^{2}$ values which bracket the range of present interest.

Since there is competition between strong attractive and strong repulsive contributors to the effective $\mathrm{NN}$ potentials at short range, it might be concluded that the nature of the potentials could depend in a very sensitive way on the details of the model or the precise values of the model parameters. For this reason effective NN potentials were evaluated for a number of different parameter sets including the second set of table 2 used earlier for a pure 3q-3q model. Although this parameter set is derived for a different model space and differs very markedly from the present set, particularly in the value of $a_{c}$, it leads to an effective NN potential qualitatively very similar to that of the present investigation, when used to evaluate the $c_{\alpha}$ and the cross-terms in the exchange kernels for the full set of $24(3 q)(q \bar{q})$ components. 


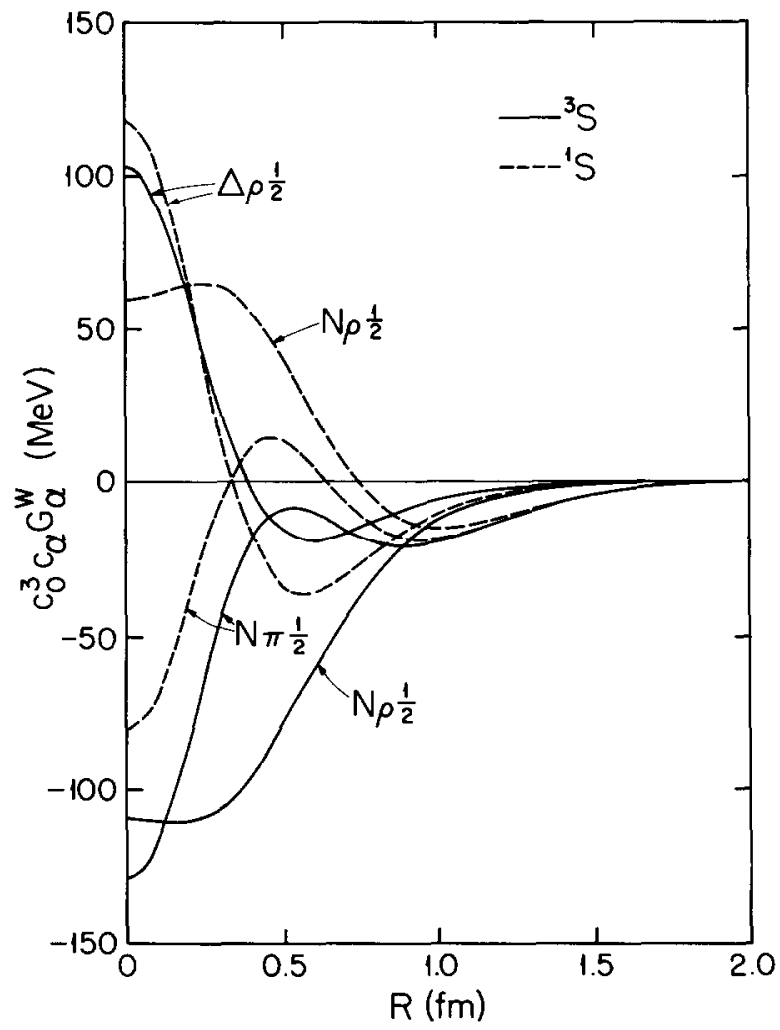

Fig. 9. The Wigner transforms for individual $(3 q)(q \bar{q})$ components. The figure shows the $c_{0}^{3} c_{\alpha} G_{\alpha}^{\mathrm{W}}\left(R ; q^{2}\right)$, with $q^{2}=0$, for the three most important contributors with $S_{12}=\frac{1}{2}$ and $(\lambda \lambda)=(00): \alpha=N \pi \frac{1}{2}, N \rho \frac{1}{2}$, and $\Delta \rho \frac{1}{2}$.

The respulsive core height at $R=0$ is still greatly reduced relative to the predictions of the simple $3 q+3 q$ model. (The core heights for $E_{\text {c.m. }}=0$ are now $20 \mathrm{MeV}$ for ${ }^{3} \mathrm{~S}$ and $0.2 \mathrm{MeV}$ for ${ }^{1} \mathrm{~S}$. For $E_{\text {c.m. }}=350 \mathrm{MeV}$ they are $247 \mathrm{MeV}$ for ${ }^{3} \mathrm{~S}$ and $229 \mathrm{MeV}$ for ${ }^{1} \mathrm{~S}$.) The strong energy dependence of the core height persists with a change in slope of $\sim 4 \%$ only, compared with the parameter set of the present investigation. The attractive parts of the effective potential are also very similar (with minima of $-35 \mathrm{MeV}$ for ${ }^{3} \mathrm{~S}$ and $-43 \mathrm{MeV}$ for ${ }^{1} \mathrm{~S}$, at $E_{\text {c.m. }}=0$, cf. fig. 5).

\section{Summary}

Quark-antiquark excitations have been incorporated into a quark model of the nucleon to study the effects of such excitations on the NN interaction. Although it is the ultimate aim of such investigations to make a more natural connection between the simple quark models and the mesonic degrees of freedom responsible for the 


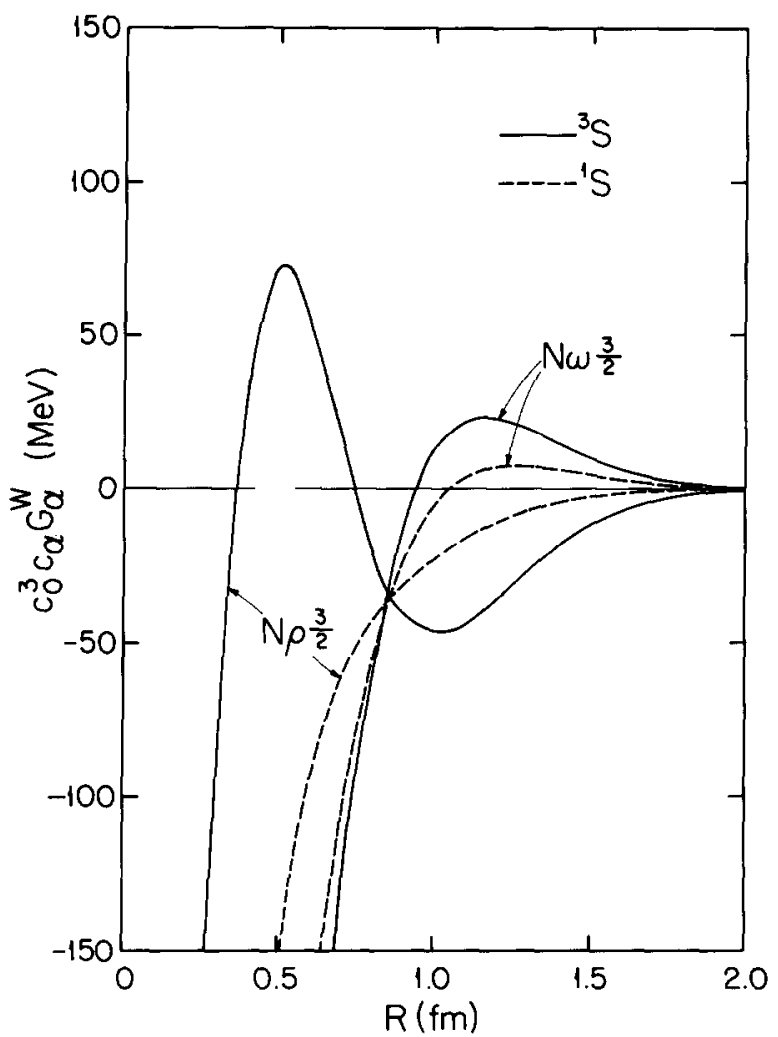

Fig. 10. Wigner transforms (as in fig. 9) for $\alpha=\mathrm{N} \omega \frac{3}{2}, \mathrm{~N} \rho \frac{3}{2}$. The $R=0$ values (beyond the range of the figure) are: for $\alpha=\mathrm{N} \omega \frac{3}{2},-1187 \mathrm{MeV}$ for ${ }^{1} \mathrm{~S}$ and $-1319 \mathrm{MeV}$ for ${ }^{3} \mathrm{~S}$; for $\alpha=\mathrm{N} \rho \frac{3}{2},-847 \mathrm{MeV}$ for ${ }^{1} \mathrm{~S}$ and $-509 \mathrm{MeV}$ for ${ }^{3} \mathrm{~S}$.

medium- and long-range parts of the $\mathrm{NN}$ interaction, the present investigation takes only a first step in that direction by introducing $(3 q)(q \bar{q})$ components into the internal wave functions of a single nucleon within the framework of the resonating group method. Although $\mathrm{q}^{4} \overline{\mathrm{q}}$ configurations for a single baryon have previously been considered ${ }^{45}$ ), these earlier studies concerned themselves with excited states of the nucleon. In the present investigation the emphasis is on the effects of such configurations on the ground state of the nucleon and in particular the two-nucleon system through their contributions to the quark exchange kernels which are used to arrive at an effective NN interaction. The qq̄ pair-creation terms inherent in the quark-gluon interaction lagrangian have been used in many earlier investigations [see e.g. ref. $\left.{ }^{46}\right)$ ]. In the present investigation the off-shell q $\bar{q}$ pair-creation terms are derived from the one-gluon-exchange diagram in the Breit approximation in complete analogy with the derivation of the $\mathrm{qq}$ and $\mathrm{q} \overline{\mathrm{q}}$ interaction potential. The parameters of the interaction, $\alpha_{\mathrm{s}}$ and $m$, and the length parameter, $b$, of the quark internal wave functions are chosen to be consistent with the experimental $\Delta-\mathrm{N}$ 


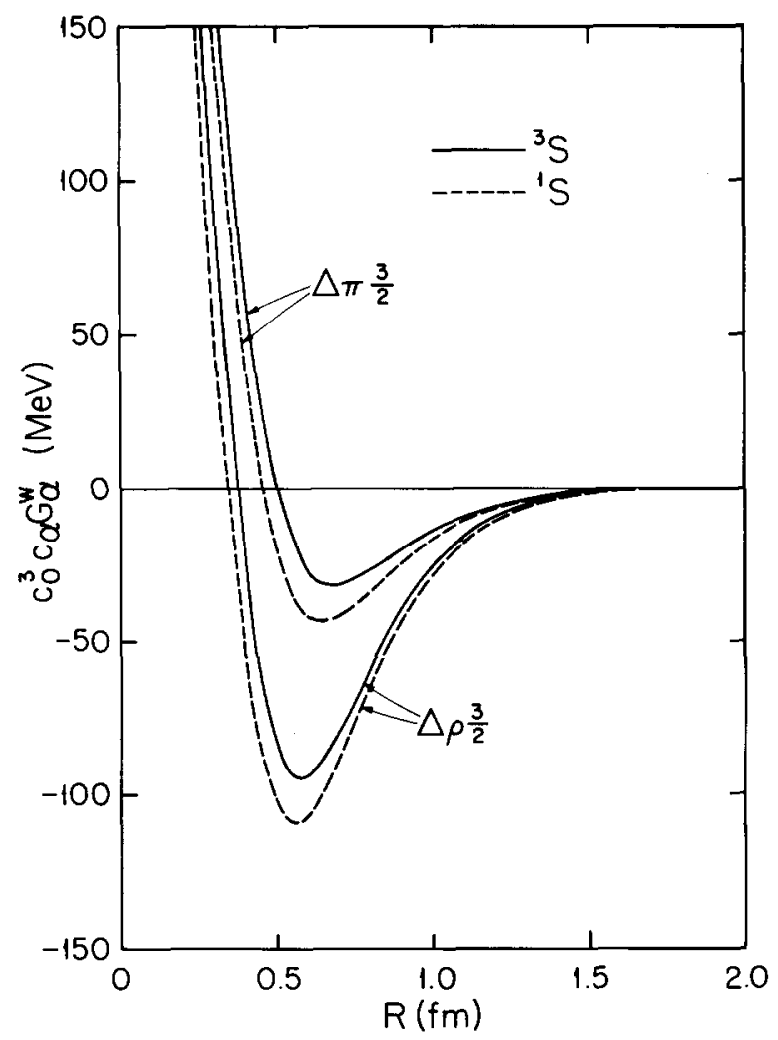

Fig. 11. Wigner transforms (as in fig. 10) for $\alpha=\Delta \pi \frac{3}{2}$ and $\Delta \rho \frac{3}{2}$. The $R=0$ values (beyond the range of the figure) are: for $\alpha=\Delta \pi \frac{3}{2},+428 \mathrm{MeV}$ for ${ }^{1} \mathrm{~S}$ and $+463 \mathrm{MeV}$ for ${ }^{3} \mathrm{~S}$; for $\alpha=\Delta \rho \frac{3}{2},+395 \mathrm{MeV}$ for ${ }^{1} \mathrm{~S}$ and $+460 \mathrm{MeV}$ for ${ }^{3} \mathrm{~S}$.

mass difference, the vector-meson-nucleon coupling constants, and the nucleon magnetic moments. Since our improved quark model for the nucleon can, however, not be expected to give a realistic picture for the extreme surface region of the nucleon we expect to underpredict the value of the proton rms radius and the pion-nucleon coupling constant. Within the above constraints, the predicted amplitudes of the $(3 q)(q \bar{q})$ components of the nucleon wave function have been shown to be quite insensitive to the precise values of the model parameters. In particular, they are very insensitive to large changes in the magnitude of the confinement potential constant which is a necessary ingredient of the model. Similar $(3 q)(q \bar{q})$ amplitudes were also obtained in a relativistic calculation using zero-rest mass MIT bag wave functions.

Phase-shift-equivalent effective local potentials are obtained from the Wigner transforms of the quark-exchange kernels through the WKB approximation. The $(3 q)(q \bar{q})$ components in the nucleon wave function lead to the following effects: (i) The repulsive core heights of the simple $3 q-3 q$ model are greatly reduced but retain 


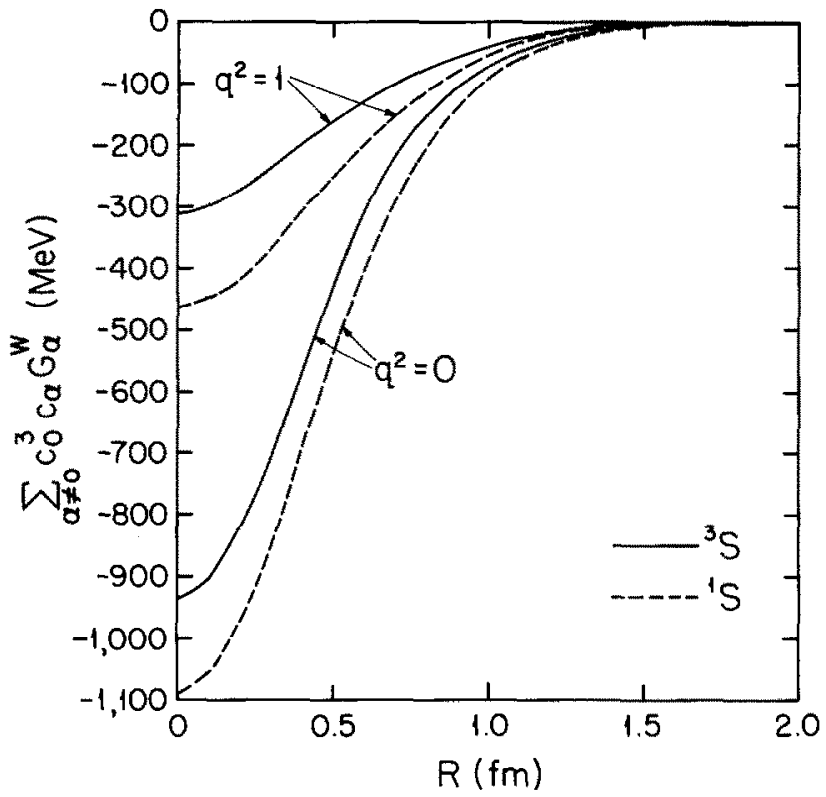

Fig. 12. The Wigner transforms; full cross-term contributions. The figure shows the summed contributions $\sum_{\alpha \neq 0} c_{0}^{3} c_{\alpha} \sigma_{\alpha}^{\mathrm{W}}\left(R ; q^{2}\right)$ for both $q^{2}=0$ and $q^{2}=+1$. (The value $q^{2}=+1$ is appropriate for $E_{\text {c.m. }}-225 \mathrm{MeV}$ as $R \rightarrow$ large; $q^{2}=0$ is appropriate as $R \rightarrow 0$ for $E_{\text {c.m. }}$ in the $100-200 \mathrm{MeV}$ range.)

their strong essentially linear energy dependence. (ii) The effective potentials have gained an attractive part in the $0.8-1.5 \mathrm{fm}$ range. However, this attraction is too weak to bind the deuteron or fit the low-energy S-wave phase shifts.

The present investigation is only a first step toward an improved quark model for the NN interaction. Even within the framework of the present model many refinements are possible. So far, all distortion effects have been neglected. The $c_{\alpha}$ which determine the $(3 q)(q \bar{q})$ amplitudes of a single nucleon should be permitted to vary with the NN separation in the full two-nucleon problem and may also be dependent on the relative momentum $\boldsymbol{K}$ of the initial two-nucleon state. More sophisticated $(\mathrm{q} \overline{\mathrm{q}})-(3 q)$ relative motion functions should be used. In particular, their

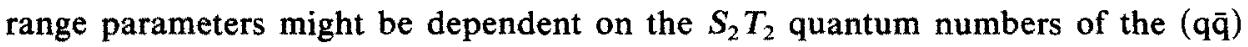
cluster. The coupling to other (closed) channels must be considered even within the framework of a $1 \hbar \omega$ oscillator model. Channels in which a pure 0 s $(3 q)(q \bar{q})$ negative-parity nucleon excited state is coupled to a 0 s (3q) cluster with a $0 p$ wave relative motion function might be important. Although the fully antisymmetrized RGM wave function of this type will have some overlap with the model space of the present investigation, the color, spin, isospin intrinsic degrees of freedom are so numerous that such channels can perhaps not be neglected. The indications are that the coupling to such channels must be considered to understand the observed 
P-wave phase shifts, in particular. Finally, more complicated $q \bar{q}$ excitations may have to be considered and a full three-cluster RGM formulation of the model may have to be used to gain a better picture of the long-range part of the NN interaction.

\section{References}

1) C. DeTar, Phys. Rev. D17 (1978) 323

2) V.G. Neudatchin, in AIP Conf. Proc. no. 47, ed. W.T.H. van Oers et al. (AIP, New York, 1978) p. 469

3) D.A. Liberman, Phys. Rev. D16 (1977) 1542

4) J.E.F.T. Ribeiro, Z. Phys. C5 (1980) 27

5) C.S. Warke and R. Shanker, Phys. Rev. C21 (1980) 2643

6) H. Toki, Z. Phys. A294 (1980) 173; Nucl. Phys. A358 (1981) 357c

7) M. Oka and K. Yazaki, Phys. Lett. 90B (1980) 41; Prog. Theor. Phys. 66 (1981) 556, 572

8) A. Faessler, F. Fernandez, G. Lübeck and K. Shimizu, Phys. Lett. 112B (1982) 201; Nucl. Phys. A402 (1983) 555

9) M. Cvetič, B. Golli, N. Mankoč-Borštnik and M. Rosina, Nucl. Phys. A395 (1983) 349

10) M. Ilarvey, Nucl. Phys. A352 (1981) 301, 326

11) M. Harvey and J. Le Tourneux, Nucl. Phys. A424 (1984) 419;

M. Harvey, J. Le Tourneux and B. Lorazo, Nucl. Phys. A424 (1984) 428

12) K. Holinde, Nucl. Phys. A415 (1984) 477

13) Y. Suzuki and K.T. Hecht, Phys. Rev. C27 (1983) 299; C28 (1983) 1458

14) Y. Suzuki and K.T. Hecht, Nucl. Phys. A420 (1984) 525

15) O. Morimatsu, S. Ohta, K. Shimizu and K. Yazaki, Nucl. Phys. A420 (1984) 573;

O. Morimatsu, K. Yazaki and M. Oka, Nucl. Phys. A424 (1984) 412

16) Y. Suzuki, Nucl. Phys. A430 (1984) 539

17) Y. Suzuki, Nucl. Phys. A444 (1985) 637

18) K. Maltman and N. Isgur, Phys. Rev. Lett. 50 (1983) 1827; Phys. Rev. D29 (1984) 952

19) J. Burger and H.M. Hofmann, Phys. Lett. 148B (1984) 25

20) M.H. Storm and A. Watt, Nucl. Phys. A408 (1983) 397; J. of Phys. G11 (1985) 217

21) I. Bender and H.G. Dosch, Fortschr. Phys. 30 (1982) 633

22) I.T. Obukhovsky, Z. Phys. A308 (1982) 253

23) S. Ohta, M. Oka, A. Arima and K. Yazaki, Phys. Lett. 119B (1982) 35; 123B (1983) 477(E)

24) D. Robson, Prog. Part. Nucl. Phys. 8 (1982) 257

25) O.W. Greenberg and H.J. Lipkin, Nucl. Phys. A370 (1981) 349

26) G.E. Brown and M. Rho, Phys. Lett. 82B (1979) 177;

V. Vento, M. Rho, E.M. Nyman, G.H. Jun and G.E. Brown, Nucl. Phys. A345 (1980) 413;

F. Myhrer, G.E. Brown and Z. Xu, Nucl. Phys. A362 (1981) 317

27) A.W. Thomas, S. Théberge and G.A. Miller, Phys. Rev. D24 (1981) 216

28) R. Friedberg and T.D. Lee, Phys. Rev. D15 (1977) 1694; D16 (1977) 1096; D18 (1978) 2623;

T.D. Lee, Phys. Rev. DI9 (1979) 1802

29) R. Goldflam and L. Wilets, Phys. Rev. D25 (1982) 1951;

H.R. Fiebig and E. Hadjimichael, Phys. Rev. D30 (1984) 181, 195;

S. Kahana, G. Ripka and Y. Soni, Nucl. Phys. A415 (1984) 351;

M.C. Birse and M.K. Banerjee, Phys. Lett. 136B (1984) 284;

Th. Köppel and M. Harvey, Phys. Rev. D31 (1985) 171;

L.S. Celenza, A. Rosenthal and C.M. Shakin, Phys. Rev. C31 (1985) 212, 232

30) E. Witten, Nucl. Phys. B223 (1983) 433;

G.S. Adkins, C.R. Nappi and E. Witten, Nucl. Phys. B228 (1983) 552;

G.S. Adkins and C.R. Nappi, Phys. Lett. 137B (1984) 251

31) M. Rho, A.S. Goldhaber and G.E. Brown, Phys. Rev. Lett. 51 (1983) 747

32) H. Horiuchi, Prog. Theor. Phys. 64 (1980) 184

33) K. Aoki and H. Horiuchi, Prog. Theor. Phys. 68 (1982) 1658, 2028 
34) A. Faessler, G. Lübeck and K. Shimizu, Phys. Rev. D26 (1982) 3280

35) Y.W. Yu and Z.Y. Zhang, Nucl. Phys. A426 (1984) 557

36) T.H. Seligman and W. Zahn, J. of Phys. G2 (1976) 79;

H. Horiuchi, Prog. Theor. Phys. 55 (1976) 1448

37) Y. Fujiwara and Y.C. Tang, University of Minnesota report, UM-RGM2 (1984)

38) Y. Suzuki, Nucl. Phys. A405 (1983) 40

39) Y. Suzuki, K.T. Hecht and H. Toki, KINAM Rev. Fis. 4 (1982) 99

40) P. Kramer and T.H. Seligman, Nucl. Phys. A136 (1969) 545; A186 (1972) 49

41) M.M. Nagels, Th.A. Rijken, J.J. deSwart, G.C. Oades, J.L. Petersen, A.C. Irving, C. Jarlskog, W. Pfeil, H. Pilkuhn and H.P. Jakob, Nucl. Phys. B147 (1979) 189

42) M. Oka and K. Yazaki, in Quarks and nuclei, ed. W. Weise (World Scientific, Singapore, 1985)

43) M. Lacombe, B. Loiseau, J.M. Richard, R. Vinh Mau, P. Pires and R. de Tourreil, Phys. Rev. D12 (1975) 1495;

R. Vinh Mau, Mesons in nuclei, vol. 1, ed. M. Rho and D. Wilkinson (North-Holland, Amsterdam, 1979) p. 152

44) M.H. MacGregor, R.A. Arndt and R.M. Wright, Phys. Rev. 182 (1969) 1714

45) D. Strottman, Phys. Rev. D20 (1979) 748

46) C.W. Wong, Prog. Part. Nucl. Phys. 8 (1982) 223;

J.F. Donoghue and E. Golowich, Phys. Rev. D15 (1977) 3421;

F.E. Close and R.R. Horgan, Nucl. Phys. B164 (1980) 413; B185 (1981) 333 